TRANSACTIONS OF THE

AMERICAN MATHEMATICAL SOCIETY

Volume 350, Number 8, August 1998, Pages 3131-3167

S 0002-9947(98)01983-7

\title{
CONVERGENCE OF MADELUNG-LIKE LATTICE SUMS
}

\author{
DAVID BORWEIN, JONATHAN M. BORWEIN, AND CHRISTOPHER PINNER
}

\begin{abstract}
We make a general study of the convergence properties of lattice sums, involving potentials, of the form occurring in mathematical chemistry and physics. Many specific examples are studied in detail. The prototype is Madelung's constant for $\mathrm{NaCl}$ :

$$
\sum_{-\infty}^{\infty} \frac{(-1)^{n+m+p}}{\sqrt{n^{2}+m^{2}+p^{2}}}=-1.74756459 \cdots,
$$
\end{abstract}

presuming that one appropriately interprets the summation proccess.

\section{INTRODUCTION}

Lattice sums of the form arising in crystalline structures - and defined precisely in the next section - have been subject to intensive study. A very good overview is available in [5] and related research may be followed up in [2, 1, 4]. These sums are highly conditional in their convergence, and the subject of how best to interpret their convergence is discussed in $[2,1,3,4]$ and the references therein.

The prototype is Madelung's constant for $\mathrm{NaCl}$ :

$$
\sum_{-\infty}^{\infty} \frac{(-1)^{n+m+p}}{\sqrt{n^{2}+m^{2}+p^{2}}}=-1.74756459 \cdots
$$

presuming that one sums over expanding cubes but not spheres, [1].

Since the analytic or numerical evaluations of such sums usually proceed by transform (and "renormalization") methods, these issues are often obscured, especially in the physical science literature. As we shall illustrate in this paper, while some general theorems are available, the precise study of convergence is a delicate and varied subject. Some of our results are unsurprising, but others are far from intuitive.

Received by the editors August 21, 1995 and, in revised form, June 24, 1996.

1991 Mathematics Subject Classification. Primary 11P21, 40A05; Secondary: 11S40, 40B05, 82D25, 30B50.

Key words and phrases. Lattice sums, zeta functions, conditional convergence, Madelung's constant, Dirichlet series, theta functions.

The first and second authors were partially supported by the Natural Sciences and Engineering Research Council of Canada. The second author also received support from the Shrum Endowment at Simon Fraser University.

(C)1998 American Mathematical Society 


\section{Preliminaries And Notation}

We shall suppose throughout that

$$
Q\left(x_{1}, \ldots, x_{k}\right):=\sum_{i=1}^{k} \sum_{j=1}^{k} \alpha_{i j} x_{i} x_{j} \in \mathbf{R}\left[x_{1}, \ldots, x_{k}\right]
$$

is a positive definite quadratic form with $\alpha_{i j}=\alpha_{j i}$. For a bounded set $C$ in $\mathbf{R}^{k}$ and a positive real number $\nu$ we understand $\nu C$ to be the set of $\left(u_{1}, \ldots, u_{k}\right) \in \mathbf{R}^{k}$ such that $\left(u_{1} / \nu, \ldots, u_{k} / \nu\right) \in C$, and we set

$$
C_{\nu}:=\nu C \cap\left(\mathbf{Z}^{k} \backslash(0, \ldots, 0)\right) .
$$

We shall chiefly be interested in $C \subset \mathbf{R}^{k}$ where $(0, \ldots, 0)$ lies in the interior of $C$, so that

$$
\lim _{\nu \rightarrow \infty} \nu C=\mathbf{R}^{k}
$$

We define the corresponding lattice sum

$$
A_{\nu}(s)=A_{\nu}(C, Q, s):=\sum_{\left(x_{1}, \ldots, x_{k}\right) \in C_{\nu}} \frac{(-1)^{x_{1}+\cdots+x_{k}}}{Q\left(x_{1}, \ldots, x_{k}\right)^{s}}
$$

and write

$$
A(s)=A(C, Q, s):=\lim _{\nu \rightarrow \infty} A_{\nu}(C, Q, s)
$$

whenever this limit exists. For the most part we shall suppress explicit reference to parameters such as $C$ and $Q$ and simply write $A(s)$ (except in Sections 4.2 and 7 where we use $A(C, Q, s)$ to emphasize the dependence upon the region $C$ ). Throughout we avoid summing over the pole at zero. We also often write $\sigma$ for Re $s$. [The literature is split as to whether to write $A(s)$ or $A(2 s)$, the latter moving the physically meaningful value from $\frac{1}{2}$ to 1.] At $s:=\frac{1}{2}$ our sums are evaluating weighted/signed potentials at the origin over points in the underlying lattice.

While we have stated our results with reference to integer lattice points, we can readily generate analogues for an arbitrary lattice $A \mathbf{Z}^{k}$ on replacing $Q(\vec{x})$ by $Q(A \vec{x})$. Notice that a convex body will be mapped to a convex body by the matrix $A^{-1}$.

Our key result is to show that $A(s)$ exists and is analytic at least down to Re $s>(k-1) / 2$ for all reasonably shaped regions $C$ (and hence that the limit is independent of the shape of $C$ chosen in that range). In fact, as the next section shows, the same is true if we replace the " $(-1)^{x_{1}+\cdots+x_{k}}$ " by a function $q\left(x_{1}, \ldots, x_{k}\right)$ exhibiting a similar degree of cancellation when summed over one of the $x_{i}$.

In Section 4 we examine in detail the question of convergence for $\operatorname{Re} s \leq(k-1) / 2$ when $C$ is an (appropriate) ellipse in $\mathbf{R}^{k}$ or $C$ is an arbitrary polygon in $\mathbf{R}^{2}$ or $\mathbf{R}^{3}$ with rational vertices or $C$ is a $k$-dimensional rectangle (showing that in the latter case convergence actually holds for all $\operatorname{Re} s>0)$. In Section 5 we give very explicit formulae when $Q(x, y):=x^{2}+P y^{2}$ for certain $P$ (particularly for $P=3$ or 7 ), and $C$ is the corresponding ellipse $x^{2}+P y^{2} \leq 1$. Several other examples are detailed in Section 6. Finally in Section 7 , when $Q(x, y):=x^{2}+y^{2}$, we demonstrate directly the existence and equivalence of the limits at $s=1$ (the most analytically pliable value) for $C$ a circle, rectangle, or diamond. Since many of the proofs are lengthy and technical, we have chosen to postpone the majority of them until Section 8 . 


\section{A Convergence Theorem for General Regions}

Let $C$ be a bounded set in $\mathbf{R}^{k}$ containing $(0, \ldots, 0)$ in its interior and let $Q\left(x_{1}, \ldots, x_{k}\right)$ be a positive definite quadratic form in $\mathbf{R}\left[x_{1}, \ldots, x_{k}\right]$. Let $q: \mathbf{Z}^{k} \rightarrow$ $\mathbf{R}$, and let

$$
A_{\nu}(s)=A_{\nu}(C, Q, q, s):=\sum_{\left(x_{1}, \ldots, x_{k}\right) \in C_{\nu}} \frac{q\left(x_{1}, \ldots, x_{k}\right)}{Q\left(x_{1}, \ldots, x_{k}\right)^{s}} .
$$

We may now state our basic result.

Theorem 1. Let $\chi_{\nu}$ be the characteristic function of $C_{\nu}$, and suppose that

$$
w_{\nu}\left(j_{1}, \ldots, j_{k-1}, m\right):=\sum_{l=-\infty}^{m} q\left(j_{1}, \ldots, j_{k-1}, l\right) \chi_{\nu}\left(j_{1}, \ldots, j_{k-1}, l\right)
$$

is uniformly bounded for all integers $j_{1}, \ldots, j_{k-1}, m$ and all positive $\nu$. Then

$$
A(s)=A(C, Q, q, s):=\lim _{\nu \rightarrow \infty} A_{\nu}(s)
$$

exists and is analytic in the region $\sigma:=\operatorname{Re} s>\frac{1}{2}(k-1)$.

This is the most that we can in general say, as can be seen by taking $C$ to be (for example) the $l_{1}$ ball in $\mathbf{R}^{k}$ :

Theorem 2. Suppose that $q\left(x_{1}, \ldots, x_{k}\right):=(-1)^{x_{1}+\cdots+x_{k}}$, and that $C$ is the $k$ dimensional diamond $a_{1}\left|x_{1}\right|+\cdots+a_{k}\left|x_{k}\right| \leq c$ where the $a_{1}, \ldots, a_{k}, c \in \mathbf{N}$ with $d=\operatorname{gcd}\left(a_{1}, \ldots, a_{k}\right)$ and all the $a_{i} / d$, are odd. Then $A_{\nu}\left(\frac{1}{2}(k-1)\right)$ does not tend to a limit as $\nu \rightarrow \infty$.

Theorem 1 shows that in $\mathbf{R}^{2}$ the limit is well defined for $\sigma>\frac{1}{2}$, and in $\mathbf{R}^{3}$ for $\sigma>1$, for any sensible region and any reasonable $q$. We make this precise in the next corollary. We say that a region $C$ in $\mathbf{R}^{k}$ is convex in the ith variable if whenever the points $\left(x_{1}, \ldots, x_{i}^{\prime}, \ldots, x_{k}\right)$ and $\left(x_{1}, \ldots, x_{i}^{\prime \prime}, \ldots, x_{k}\right)$ are in $C$ then so also is the segment joining them.

Corollary 1. Suppose that $q\left(x_{1}, \ldots, x_{i}, \ldots, x_{k}\right)$ is bounded over $\mathbf{Z}^{k}$ and is periodic with period $M$ in one variable, $x_{i}$ say, with

$$
\sum_{x_{i}=1}^{M} q\left(x_{1}, \ldots, x_{k}\right)=0 .
$$

Suppose further that $C$ is bounded, contains $(0, \ldots, 0)$ in its interior and is convex in the ith variable. Then the conclusions of Theorem 1 hold.

Indeed, many highly non-convex regions still satisfy Theorem 1 . We will refer to a vertically convex region in $\mathbf{R}^{k}$ as being one in which the final coordinate exhibits convexity.

We now focus on sums over specific regions; showing that in some cases convergence can continue well below $\sigma=(k-1) / 2$. 


\section{Specific Regions}

4.1. Lattice Sums over Sympathetic Ellipses. Given a positive definite quadratic form $Q\left(x_{1}, \ldots x_{k}\right)=\sum_{i=1}^{k} \sum_{j=1}^{k} \alpha_{i j} x_{i} x_{j} \in \mathbf{Z}\left[x_{1}, \ldots, x_{k}\right]$ with $\alpha_{i j}=\alpha_{j i}$ and a function $q: \mathbf{Z}^{k} \rightarrow \mathbf{R}$, we define the arithmetic function

$$
r(n, Q, q):=\sum_{\substack{Q(\vec{x})=n \\ \vec{x} \in \mathbf{Z}^{k}}} q(\vec{x}) .
$$

In particular, when our quadratic form has integer coefficients and we sum over the lattice points in appropriate ellipses, $Q\left(x_{1}, \ldots, x_{k}\right) \leq \nu$, we can replace the $k$-dimensional lattice sum by a Dirichlet series

$$
A_{\nu}(s)=A_{\nu}(Q, q, s):=\sum_{\substack{Q(\vec{x}) \leq \nu \\ \vec{x} \in \mathbf{Z}^{k} \backslash \overrightarrow{0}}} \frac{q(\vec{x})}{Q(\vec{x})^{s}}=\sum_{1 \leq n \leq \nu} \frac{r(n, Q, q)}{n^{s}},
$$

and decide when the limit

$$
A(s)=A(Q, q, s):=\lim _{\nu \rightarrow \infty} \sum_{1 \leq n \leq \nu} \frac{r(n, Q, q)}{n^{s}}
$$

exists by examining the sums

$$
S_{0}(x)=S_{0}(Q, q, x):=\sum_{0 \leq n \leq x} r(n, Q, q) .
$$

We recall the formula (see Hardy [6, Theorem 7]) for the abscissa of convergence $\sigma_{0}>0$ of such a Dirichlet series;

$$
\sigma_{0}=\limsup _{x \rightarrow \infty} \frac{\log \left|S_{0}(x)\right|}{\log x} .
$$

That is (see Hardy [6, Theorem 1]), $A(s)$ will exist for all $\operatorname{Re} s>\sigma_{0}$ and fail to exist for all $\operatorname{Re} s<\sigma_{0}$.

We show that (at least for periodic $q$ with suitable cancellation when summed) convergence over these ellipses always extends below $\sigma=(k-1) / 2$ :

Theorem 3. Suppose that $q\left(x_{1}, \ldots, x_{k}\right)$ is periodic with period $M$ in each of the $x_{i}$. If

$$
\sum_{r_{1}=1}^{M} \cdots \sum_{r_{k}=1}^{M} q\left(r_{1}, \ldots, r_{k}\right)=0,
$$

then the abscissa of convergence $\sigma_{0}$ satisfies

$$
0<\sigma_{0} \leq \begin{cases}23 / 73, & \text { if } k=2, \\ 25 / 34, & \text { if } k=3 \\ k / 2-1, & \text { if } k \geq 4\end{cases}
$$

If

$$
\sum_{r_{1}=1}^{M} \cdots \sum_{r_{k}=1}^{M} q\left(r_{1}, \ldots, r_{k}\right) \neq 0,
$$

then the abscissa of convergence $\sigma_{0}=k / 2$. 
The theorem follows easily from old work of Landau and Walfisz (for $k \geq 4$ ), and more recent bounds of Krätzel \& Nowak (for $k=3$ ) and Huxley (for $k=2$ ), on the error in approximating the number of lattice points in an ellipsoid by its volume. When $k \geq 4$ these bounds cannot in general be improved:

Theorem 4. If $q\left(x_{1}, \ldots, x_{k}\right):=(-1)^{x_{1}+\cdots+x_{k}}$ and $Q\left(x_{1}, \ldots, x_{k}\right):=a_{1} x_{1}^{2}+\cdots+$ $a_{k} x_{k}^{2}$, where the $a_{i}$ are all odd positive integers, then for all $k \geq 2$ the limit $A(s)$ does not exist for any $\operatorname{Re} s \leq(k / 2)-1$.

When $k=2$ or 3 one expects the correct upper bounds to be $1 / 4$ and $1 / 2$ respectively. From this last theorem, $1 / 2$ would certainly be sharp when $k=3$. In fact we show that for very general $Q$ and $q$ we have the lower bound $\sigma_{0} \geq(k-1) / 4$, so that when $k=2$ or 3 we usually do indeed have the lower bounds $1 / 4$ and $1 / 2$ :

Theorem 5. Suppose that $q\left(x_{1}, \ldots, x_{k}\right)$ is periodic in all of the $x_{i}$ with period $M$ and

$$
\sum_{r_{1}=1}^{M} \cdots \sum_{r_{k}=1}^{M} q\left(r_{1}, \ldots, r_{k}\right)=0
$$

If $r(n, Q, q)$ (and hence $\left.A_{\nu}(s)\right)$ is not identically 0 , then

$$
\sigma_{0} \geq \frac{1}{4}(k-1)
$$

Notice that there certainly will be cases with $A_{\nu}(s)$ identically zero (with therefore no lower bound on $\left.\sigma_{0}\right)$; indeed for any $M \neq 2$ we can always construct nontrivial periodic $q(\vec{x})$ with $q(-\vec{x})=-q(\vec{x})$ and hence, by symmetry, the $r(n, Q, q)$ zero for all $n$ and any $Q$.

The proof of Theorem 5 will use a technique of Landau to show the existence of a constant $c_{0}=c_{0}(q, Q)>0$ such that

$$
\left|S_{0}(x)\right|>c_{0} x^{(k-1) / 4}
$$

for infinitely many integers $x$. The method requires some additional notation:

Given a positive definite quadratic form $Q\left(x_{1}, \ldots, x_{k}\right)=\sum_{i=1}^{k} \sum_{j=1}^{k} \alpha_{i j} x_{i} x_{j}$ in $\mathbf{Z}[x]$ with $\alpha_{i j}=\alpha_{j i}$, we let $D$ denote the determinant

$$
D:=\left|\begin{array}{ccc}
\alpha_{11} & \ldots & \alpha_{1 k} \\
\vdots & & \vdots \\
\alpha_{k 1} & \ldots & \alpha_{k k}
\end{array}\right|
$$

and define the positive definite adjoint quadratic form $Q^{*}\left(x_{1}, \ldots, x_{k}\right)$ in $\mathbf{Z}[x]$

$$
Q^{*}\left(x_{1}, \ldots, x_{k}\right):=\sum_{i=1}^{k} \sum_{j=1}^{k} \frac{\partial D}{\partial \alpha_{i j}} x_{i} x_{j}
$$

Notice that $Q^{* *}(\vec{x})=Q(\vec{x})$ and that when $k=2$ we have $Q^{*}(x, y)=Q(-y, x)$. We suppose that $q\left(x_{1}, \ldots, x_{k}\right)$ is periodic in each of the $x_{i}$ with period $M$ and define the periodic weight function

$$
\lambda_{q}(\vec{x}):=\sum_{r_{1}=1}^{M} \cdots \sum_{r_{k}=1}^{M} q\left(r_{1}, \ldots, r_{k}\right) \cos \left(\frac{2 \pi}{M}\left(r_{1} x_{1}+\cdots+r_{k} x_{k}\right)\right)
$$


and set

$$
r^{*}(n)=r^{*}(n, Q, q):=\sum_{\substack{\vec{x} \in \mathbf{Z}^{k} \\ Q^{*}(\vec{x})=n}} \lambda_{q}(\vec{x}) .
$$

For the $q(\vec{x})$ of interest the involved looking expression for $r^{*}(n)$ often simplifies. For example, when

$$
q\left(x_{1}, \ldots, x_{k}\right):=(-1)^{x_{1}+\cdots+x_{s}}
$$

for some $1 \leq s \leq k$, we have

$$
r^{*}(n)=2^{k} \sum_{\begin{array}{c}
Q^{*}\left(x_{1}, \ldots, x_{k}\right)=n \\
x_{1}, \ldots, x_{s} \text { odd } \\
x_{s+1}, \ldots, x_{k} \text { even }
\end{array}} 1
$$

We remark that $r(n, Q, q)$ is identically zero if and only if $r^{*}(n)$ is identically zero.

As with the classical circle problem, our proof relies on the ability to write the sum $S_{0}(x)$ in terms of Bessel functions $J_{\nu}(x)$;

$$
\sum_{0 \leq n \leq x}{ }^{*} r(n, Q, q)=\frac{(\sqrt{D} M)^{\frac{1}{2}(k-2)}}{M} x^{k / 4} \sum_{n=1}^{\infty} \frac{r^{*}(n)}{n^{k / 4}} J_{k / 2}\left(\frac{2 \pi}{M} \sqrt{\frac{n x}{D}}\right)
$$

where $\sum^{*}$ denotes that if $x$ is an integer the last term receives only half weighting $\frac{1}{2} r(x, Q, q)$. In fact we shall actually use more assuredly convergent integrated forms of this.

As one consequence of the proof, defining

$$
S_{\nu}(x):=\frac{1}{\nu !} \sum_{0 \leq n \leq x}(x-n)^{\nu} r(n, Q, q)
$$

and setting

$$
\mathcal{B}_{\nu}^{(\rho)}(s):=A_{\nu}(s)-\sum_{i=0}^{\rho-1} \frac{\Gamma(s+i)}{\Gamma(s)}\left(\frac{S_{i}(\nu)}{\nu^{s+i}}-\frac{q(\overrightarrow{0})}{i !}\right)+\frac{\Gamma(s+\rho)}{\Gamma(s)} \frac{q(\overrightarrow{0})}{\rho !},
$$

it will be apparent that for any positive integer $\rho>(k-1) / 2$ we can write

$$
\mathcal{B}_{\nu}^{(\rho)}(s)=\mathcal{A}^{(\rho)}(s)+O\left(\nu^{-\frac{1}{2}\left(\rho-\frac{(k-1)}{2}+2 \sigma\right)}\right),
$$

where $\mathcal{A}^{(\rho)}(s)$ is analytic in the larger region

$$
\left\{\sigma>-\frac{1}{2}\left(\rho-\frac{1}{2}(k-1)\right)+\varepsilon,|s|<K\right\}
$$

for any fixed $K$ and $\varepsilon>0$, and possesses the representation

$$
\mathcal{A}^{(\rho)}(s)=\frac{2^{k / 2+\rho} \pi^{k / 2-1}}{M} \frac{\Gamma(s+\rho+1)}{\Gamma(s)}\left(\frac{\sqrt{D} M}{2 \pi}\right)^{k-2 s-1} \sum_{n=1}^{\infty} \frac{r^{*}(n)}{n^{k / 2-s}} F_{\rho}\left(\frac{2 \pi}{M} \sqrt{\frac{n}{D}}, s\right)
$$

with

$$
F_{\rho}(z, s):=z^{2 s-k} \int_{z}^{\infty} v^{k / 2-1-\rho-2 s} J_{k / 2+\rho}(v) d v .
$$

When $k=3$ we note some similarity to the relation of Buhler \& Crandall $[4,(1.5)]$. 
Finally, for $k \geq 4$, the work of Novák enables us to extend the optimal bound $\sigma_{0}=k / 2-1$ of Theorem 4 to a broader (if less easily described) class of $q$ and $Q$.

We shall say that we are in the non-singular case if, as before $q\left(x_{1}, \ldots, x_{k}\right)$ is periodic in all of the $x_{i}$ with period $M$ and

$$
\sum_{r_{1}=1}^{M} \cdots \sum_{r_{k}=1}^{M} q\left(r_{1}, \ldots, r_{k}\right)=0,
$$

and moreover there exist integers $h$ and $l>0$ with $(h, l)=1$ and

$$
\sum_{r_{1}=1}^{l M} \cdots \sum_{r_{k}=1}^{l M} q\left(r_{1}, \ldots, r_{k}\right) \exp \left(-\frac{2 \pi i h}{l} Q\left(r_{1}, \ldots, r_{k}\right)\right) \neq 0 .
$$

Note that any such $l$ necessarily has $(l, M) \neq 1$.

Theorem 6. For non-singular pairs of $q\left(x_{1}, \ldots, x_{k}\right)$ and $Q\left(x_{1}, \ldots, x_{k}\right)$ we have

$$
\sigma_{0} \geq \frac{k}{2}-1
$$

We shall show in the following corollary that any $q\left(x_{1}, \ldots, x_{k}\right)$ of the form

$$
q\left(x_{1}, \ldots, x_{k}\right):=(-1)^{x_{1}+\cdots+x_{s}}, \quad 1 \leq s \leq k,
$$

is non-singular for all $Q\left(x_{1}, \ldots, x_{k}\right)$, so that we certainly recover Theorem 4 by this approach (of course the proof of Theorem 6 will be much less elementary than that of Theorem 4):

Corollary 2. If $q\left(x_{1}, \ldots, x_{k}\right)$ is of the form

$$
q\left(x_{1}, \ldots, x_{k}\right):=\exp \left(\frac{2 \pi i}{M}\left(a_{1} x_{1}+\cdots+a_{k} x_{k}\right)\right)
$$

for some integers $a_{i}$, then the case is non-singular and

$$
\sigma_{0} \geq \frac{k}{2}-1
$$

for all positive definite quadratic forms $Q\left(x_{1}, \ldots, x_{k}\right)$ in $\mathbf{Z}\left[x_{1}, \ldots, x_{k}\right]$.

When $q\left(x_{1}, \ldots, x_{k}\right)$ takes the special form $\exp \left(\frac{2 \pi i}{M}\left(a_{1} x_{1}+\cdots+a_{k} x_{k}\right)\right)$ if the $x_{i} \equiv b_{i}\left(\bmod M_{i}\right)$ for some integers $a_{i}, b_{i}$ and $M_{i}$ (with $\left.M_{i} \mid M\right)$ and zero otherwise, Walfisz [18] has shown (see Novák [15]) that in the singular case the upper bound can be lowered to $\sigma_{0} \leq k / 4-1 / 10$ for $k>4$. Thus this division into singular and non-singular cases (although not immediately digestible) is probably the correct characterization as regards the abscissa, and Theorem 5 is conceivably the best general lower bound.

4.2. Lattice Sums over Polygons in $\mathbf{R}^{2}$ and $\mathbf{R}^{3}$. In this section we restrict ourselves to the usual weight function $q\left(x_{1}, \ldots, x_{k}\right):=(-1)^{x_{1}+\cdots+x_{k}}$. We write $A(C, Q, s)$ for the corresponding lattice sum, rather than merely $A(s)$, to emphasize the dependence here upon the region $C$ (the $Q$ dependence is of use in the proof of Theorem 8). For polygons in $\mathbf{R}^{2}$ with rational vertices we show that either convergence occurs for all Re $s>0$ or else convergence fails at $s=1 / 2$. Moreover we give an explicit and somewhat surprising diophantine criterion for deciding this based on the parity of the numerators and denominators of the slopes of the lines making up the perimeter. 
Theorem 7. Suppose $P$ in $\mathbf{R}^{2}$ is a closed polygon with rational vertices whose sides lie on the lines $a_{i} x-b_{i} y=c_{i}$, where $a_{i}, b_{i}, c_{i} \in \mathbf{Z}$ with $\operatorname{gcd}\left(a_{i}, b_{i}, c_{i}\right)=1$ and $d_{i}=\operatorname{gcd}\left(a_{i}, b_{i}\right)$.

(i) If $a_{i} / d_{i}$ and $b_{i} / d_{i}$ are of opposite parity for all $i$, then $A(P, Q, s)$ exists and is analytic for all $\operatorname{Re} s>0$.

(ii) If $a_{i} / d_{i}$ and $b_{i} / d_{i}$ are both odd for at least one of the $i$, then $A(P, Q, s)$ exists for $\operatorname{Re} s>1 / 2$ but does not exist for any real $s \leq 1 / 2$.

If in addition $P$ is star-shaped around $(0,0)$ then, even restricting to integer $n$, the limit $\lim _{n \rightarrow \infty} A_{n}(P, Q, s)$ does not exist for any real $s \leq 1 / 2$.

We extract two simple cases for further advertisement in the next corollary:

Corollary 3. (i) If $R$ is a rectangle with rational vertices and sides parallel to the axes, then $A(R, Q, s)$ exists for $\operatorname{Re} s>0$.

(ii) If $D$ is the diamond $a|x|+b|y| \leq c$, where $a, b, c \in \mathbf{N}$ with $\operatorname{gcd}(a, b, c)=1$ and $d=\operatorname{gcd}(a, b)$, then $A(D, Q, s)$ exists for $\operatorname{Re} s>0$ if $a / d$ and $b / d$ are of opposite parity, but fails to exist for any real $s \leq 1 / 2$ if $a / d$ and $b / d$ are both odd.

We note that the last corollary allows us to observe that in the Hausdorff metric, or any other reasonable metric, the convex bodies for which convergence works for all $\sigma>0$ are dense in the convex bodies in the unit ball, as are those for which convergence is destroyed for $s=\frac{1}{2}$.

Theorem 6 follows from a more precise version that (for $\operatorname{Re} s>0$ ) reduces the problem of convergence over the polygon to the question of convergence solely along the boundary. If we define a variant of the characteristic function $\chi_{C}^{*}$ where points on the boundary of $C$ receive weight $1 / 2$, and a corresponding analogue of $A_{\nu}(P, Q, s)$,

$$
A_{\nu}^{*}(C, Q, s):=\sum_{(x, y) \in C_{\nu}}(-1)^{x+y} \frac{\chi_{\nu C}^{*}(x, y)}{Q(x, y)^{s}}
$$

then the following is true:

Proposition 1. Let $Q(x, y)=\alpha x^{2}+\beta x y+\gamma y^{2}$ be a positive definite quadratic form, and $P$ a closed polygon in $\mathbf{R}^{2}$ with rational vertices whose sides lie on the lines $a_{i} x-b_{i} y=c_{i}$, where $a_{i}, b_{i}, c_{i} \in \mathbf{Z}$ with $\operatorname{gcd}\left(a_{i}, b_{i}, c_{i}\right)=1$. If $N$ is a multiple of all the $\operatorname{gcd}\left(a_{i}, b_{i}\right)$ then

$$
A_{N}^{*}(P, Q, s)=F(P, Q, s)+O_{P, Q}\left(N^{-2 \sigma}\right),
$$

where $F(P, Q, s)$ is analytic in the whole half-plane $\sigma:=\operatorname{Re} s>0$.

Similarly in three dimensions we show that convergence either fails at $s=1$ or continues down to $s=1 / 2$.

Theorem 8. Suppose that $P$ is a three dimensional polygon containing $\overrightarrow{0}$ in its interior and star-shaped about $\overrightarrow{0}$, whose faces lie on the planes $a_{i} x+b_{i} y+c_{i} z=e_{i}$, where the $a_{i}, b_{i}, c_{i}$ and $e_{i}$ are integers with $d_{i}=\operatorname{gcd}\left(a_{i}, b_{i}, c_{i}\right)$.

(i) If every face has at least one of $a_{i} / d_{i}, b_{i} / d_{i}, c_{i} / d_{i}$ even, then $A(P, Q, s)$ exists for all $\operatorname{Re} s>1 / 2$.

(ii) If there is at least one face with $a_{i} / d_{i}, b_{i} / d_{i}, c_{i} / d_{i}$ all odd, then $A(P, Q, s)$ exists for all $\operatorname{Re} s>1$ but fails to exist for any real $s \leq 1$. 
We presume that these two and three dimensional theorems can be generalized to more dimensions. We have already seen in Theorem 2 that the result on diamonds extends naturally to higher dimensions. In the next section we show that the behaviour over squares can be similarly recaptured in arbitrary dimensions.

4.3. Sums over Rectangles. When we sum over $k$-dimensional rectangles we are able to show that in general convergence holds for all Re $s>0$. More precisely, given an $\vec{m}=\left(m_{1}, \ldots, m_{k}\right)$ in $\mathbf{N}^{k}$ we define the lattice sum over the corresponding rectangle:

$$
A_{\vec{m}}(s):=\sum_{n_{1}=-m_{1}}^{m_{1}} \cdots \sum_{n_{k}=-m_{k}}^{m_{k}} \frac{q\left(n_{1}, \ldots, n_{k}\right)}{Q\left(n_{1}, \ldots, n_{k}\right)^{s}}
$$

(where as usual the pole $\left(n_{1}, \ldots, n_{k}\right)=(0, \ldots, 0)$ is omitted), and set

$$
A(s):=\lim _{\min m_{i} \rightarrow \infty} A_{\vec{m}}(s)
$$

whenever that limit exists.

Theorem 9. If the sums

$$
\sum_{n_{1}=-j_{1}}^{l_{1}} \cdots \sum_{n_{k}=-j_{k}}^{l_{k}} q\left(n_{1}, \ldots, n_{k}\right)
$$

are uniformly bounded for all integers $j_{1}, \ldots, j_{k}, l_{1}, \ldots, l_{k}$, then the limit $A(s)$ exists and is analytic for all $\sigma>0$.

4.4. $l_{p}$-Balls: an Open Question. It is natural to make an examination of $l_{p}$ sums for $p \in \mathbf{N}, p>2$. That is, for example when $k=2, C:=\left\{(x, y):|x|^{p}+|y|^{p} \leq\right.$ $1\}$. We are able to state (see [8]) asymptotically sharp expressions for the number of lattice points in these regions:

$$
\sum_{\substack{|n|^{p}+|m|^{p} \leq x \\(n, m) \in \mathbf{Z}^{2} \backslash(0,0)}} 1=\left(\frac{2 \Gamma^{2}\left(p^{-1}\right)}{p \Gamma\left(2 p^{-1}\right)}\right) x^{2 / p}+O\left(x^{(1 / p)-\left(1 / p^{2}\right)}\right), \quad p \geq 3 .
$$

Unfortunately the $l_{p}$ ball and the underlying ellipse seem highly "unsympathetic", and we leave as an open question what one can provide in the way of lower or upper bounds on $\sigma_{0}$ in this case (the most natural example to consider being $p=4$ ).

\section{Some Analytic Continuations}

We shall write $\alpha(s)$ for the alternating zeta-function

$$
\alpha(s):=\sum_{n=1}^{\infty} \frac{(-1)^{n+1}}{n^{s}}
$$

and $L_{ \pm d}(s)$ for the $L$-series

$$
L_{ \pm d}(s):=\sum_{n=1}^{\infty}( \pm d \mid n) n^{-s}
$$

where $(d \mid n)$ is the Kronecker (generalized Legendre) symbol. 
When $Q(x, y):=x^{2}+p y^{2}$ with $p=3$ or 7 , we can write down an analytic continuation of our lattice sum in terms $\alpha(s)$ and $L_{-p}(s)$ :

$$
\begin{aligned}
\sum_{(x, y) \in \mathbf{Z}^{2} \backslash(0,0)} \frac{(-1)^{x+y}}{\left(x^{2}+3 y^{2}\right)^{s}} & =-2\left(1+2^{1-s}\right) \alpha(s) L_{-3}(s), \\
\sum_{(x, y) \in \mathbf{Z}^{2} \backslash(0,0)} \frac{(-1)^{x+y}}{\left(x^{2}+7 y^{2}\right)^{s}} & =-2 \alpha(s) L_{-7}(s),
\end{aligned}
$$

resembling the representation (see Glasser-Zuckerman [5])

$$
\sum_{(x, y) \in \mathbf{Z}^{2} \backslash(0,0)} \frac{(-1)^{x+y}}{\left(x^{2}+y^{2}\right)^{s}}=-4 \alpha(s) L_{-4}(s) .
$$

These will arise from our ability to write

$$
\sum_{(x, y) \in \mathbf{Z}^{2} \backslash(0,0)} \frac{1}{Q_{p}(x, y)^{s}}=u \zeta(s) L_{-p}(s)
$$

for the quadratic form

$$
Q_{p}(x, y):=\left(\frac{p+1}{4}\right) x^{2}+\left(\frac{p-1}{2}\right) x y+\left(\frac{p+1}{4}\right) y^{2}
$$

when $p=3,7,11,19,43,67$ or 163 (the primes for which $\mathbf{Q}(\sqrt{-p})$ is a unique factorization domain), where $u=6$ if $p=3$ and 2 otherwise (the number of units in $\mathbf{Q}(\sqrt{-p})$ ). Unfortunately it is not clear how to insert a $(-1)^{x+y}$ into (5) or how to replace the $Q_{p}(x, y)$ in those sums by $x^{2}+p y^{2}$ other than when $p=3$ or 7 .

Many sums of this type have been obtained by Glasser, Zucker and Robertson $[5,21,22]$ for forms whose discriminant is disjoint (i.e. have one form per genus):

$$
\begin{gathered}
\sum_{(x, y) \in \mathbf{Z}^{2} \backslash(0,0)} \frac{(-1)^{x+y}}{\left(x^{2}+P y^{2}\right)^{s}}=-2^{1-t} \sum_{\mu \mid P}\left(1-(2 \mid \mu) 2^{1-s}\right) L_{ \pm \mu} L_{\mp 4 P / \mu} \\
\sum_{(x, y) \in \mathbf{Z}^{2} \backslash(0,0)} \frac{(-1)^{x}}{\left(x^{2}+2 P y^{2}\right)^{s}}=-2^{1-t} \sum_{\mu \mid P}\left(1-(2 \mid \mu) 2^{1-s}\right) L_{ \pm \mu} L_{\mp 8 P / \mu}
\end{gathered}
$$

where $L_{ \pm \mu}$ is taken such that $\mu \equiv \pm 1(\bmod 4)$ and where $P$ are certain square-free ( $\equiv 1(\bmod 4)$ in the second case) numbers with $t$ prime factors. The appropriate $P<10,000$ are $P=5,13,21,33,37,57,85,93,105,133,165,177,253,273$, $345,357,385,1365$ and $P=1,3,5,11,15,21,29,35,39,51,65,95,105,165$, 231 respectively (in the latter case similar representations can be shown to hold for $\left.x^{2}+8 P y^{2}\right)$. Zucker and Robertson also obtain results for the forms $x^{2}+P y^{2}$, $x^{2}+4 P y^{2}$ and $x^{2}+16 P y^{2}$ when $P=3,7$ or 15 (thus including the continuations of our sums above, although their approach is different from ours).

\section{Some Specific Sums}

We have now obtained very explicit if quite contrasting results regarding the range of convergence from the above theorems for shapes such as circles, diamonds and squares. We continue with some related examples.

(a) It was shown in Borwein-Borwein-Taylor $[1, \S \mathrm{VI}]$ that study of Madelung's constant for a two-dimensional hexagonal lattice sum with ions of alternating unit 
charge placed at the points of a lattice with basis vectors $(1,0)$ and $(1 / 2, \sqrt{3} / 2)$ leads naturally to sums of the form

$$
\sum_{(n, m) \in C_{N}} \frac{q(n, m)}{\left(n^{2}+n m+m^{2}\right)^{s}}
$$

where

$$
q(n, m):=\frac{4}{3} \sin \left(\frac{2}{3}(n+1) \pi\right) \sin \left(\frac{2}{3}(m+1) \pi\right)-\frac{4}{3} \sin \left(\frac{2}{3} n \pi\right) \sin \left(\frac{2}{3}(m-1) \pi\right) .
$$

Applying the above theorems, it is clear, on splitting $q$ up appropriately, that when Re $s>1 / 2$ convergence occurs in such a sum when the lattice points are summed over any vertically convex set, that for expanding rectangles convergence holds for all $\operatorname{Re} s>0$, and that on summing over expanding ellipses $m^{2}+m n+n^{2} \leq N$ convergence fails at some point between $\sigma=1 / 4$ and $\sigma=23 / 73$. Notice that (in the notation of section 4.1)

$$
r^{*}(n)=\frac{9}{2} \sum_{\substack{x^{2}+x y+y^{2}=n \\ x \equiv y \neq 0(\bmod 3)}} 1 .
$$

The sum was also shown to possess a similar analytic continuation to those mentioned in Section 5:

$$
h_{2}(s)=3\left(1-3^{1-s}\right)\left(1-2^{1-s}\right)^{-1} \alpha(s) L_{-3}(s) .
$$

(b) In [5] sums like

$$
c_{2}(s):=\sum_{(j, k) \in C_{\nu}} \frac{(-1)^{j}}{\left(j^{2}+k^{2}\right)^{s}}
$$

and

$$
c_{3}(s):=\sum_{(j, k, p) \in C_{\nu}} \frac{(-1)^{j}}{\left(j^{2}+k^{2}+p^{2}\right)^{s}}
$$

are discussed and are again covered by our previous analysis, with convergence over vertically convex sets holding for all $\sigma>1 / 2$ (respectively 1 ) and over circles (respectively spheres) failing at some point between $1 / 4$ and $23 / 73$ (respectively $1 / 2$ and $3 / 4$ ). After a change of variables $j=j^{\prime}+k$ (respectively $j=j^{\prime}+k+p$ ) convergence over squares (respectively cubes) can be seen (from $\S 4.2$ ) to fail at $1 / 2$ (respectively 1). However (from §4.3) convergence does hold for all $\sigma>0$ over certain other parallelepipeds.

(c) Let $r_{N}(n)$ denote the number of representations of $n$ as a sum of $N$ squares (counting permutation and sign). Then the Dirichlet sum

$$
b_{N}(s):=\sum_{n=1}^{\infty}(-1)^{n} \frac{r_{N}(n)}{n^{s}}
$$

is a special case of the sums covered in Theorem 3 and Theorem 4. In particular, it follows from $[2$, p. 290] that

$$
b_{4}(s):=-8 \alpha(s) \alpha(s-1) .
$$

Notice that from our prior analysis the abscissa of convergence is exactly equal to 1. 
Correspondingly, $b_{3}\left(\frac{1}{2}\right)$ is Madelung's constant for sodium chloride. Theorem 9 recovers the fact that the limit is taken appropriately if we sum over hypercubes. Theorem 2 shows that diamonds fail below 1 and Theorem 3 and Theorem 4 show that the exact abscissa for convergence over spheres lies between $\frac{1}{2}$ and $\frac{3}{4}$. Theorem 4 recaptures the argument in [1] that shows that convergence fails at $\frac{1}{2}$, and we would conjecture that convergence obtains for $\sigma>\frac{1}{2}$.

\section{Direct Analysis at $s=1$}

In the most basic case $Q(x, y):=x^{2}+y^{2}$ and $s:=1$ one can directly establish that the limit $A(C, Q, 1)=-\pi \log 2$ when $C$ is either the square $|x| \leq 1,|y| \leq 1$, or the diamond $|x|+|y| \leq 1$, or the circle $x^{2}+y^{2} \leq 1$ (i.e., summing over the standard $l_{p}$ balls for $\left.p=1,2, \infty\right)$, as we show below.

Observe that, when $C$ is the above unit circle,

$$
A(C, Q, 1)=\lim _{n \rightarrow \infty} \sum_{0<i^{2}+j^{2} \leq n^{2}} \frac{(-1)^{i+j}}{i^{2}+j^{2}}=\sum_{k=1}^{\infty} \frac{(-1)^{k} r_{2}(k)}{k},
$$

where $r_{2}(k)$ is the number of ways of expressing $k$ as the sum of two squares of integers in $\mathbf{Z}$. Let

$$
S_{n}:=\sum_{k=1}^{n} \frac{(-1)^{k} r_{2}(k)}{k}, \quad A_{n}:=\sum_{k=1}^{n} r_{2}(k) .
$$

Then

$$
S_{2 n}=2 \sum_{k=1}^{n} \frac{r_{2}(2 k)}{2 k}-\sum_{k=1}^{2 n} \frac{r_{2}(k)}{k}=-\sum_{k=n+1}^{2 n} \frac{r_{2}(k)}{k},
$$

since $r_{2}(2 k)=r_{2}(k)$, because $k=i^{2}+j^{2} \Leftrightarrow 2 k=(i+j)^{2}+(i-j)^{2}$. It follows by partial summation that

$$
S_{2 n}=-\sum_{k=n}^{2 n} \frac{A_{k}}{k(k+1)}-\frac{A_{2 n}}{2 n+1}+\frac{A_{n}}{n} .
$$

Further, we are familiar with the fact that

$$
\frac{A_{n}}{n}=\pi+\epsilon_{n} \text { with } \epsilon_{n}=O\left(n^{-1 / 2}\right) \text {. }
$$

Hence

$$
S_{2 n}=-\pi \sum_{k=n}^{2 n} \frac{1}{k+1}-\sum_{k=n}^{2 n} \frac{\epsilon_{k}}{k+1}+o(1)=-\pi \log 2+o(1) \text { as } n \rightarrow \infty .
$$

Finally, since $r_{2}(n)=O\left(n^{1 / 2}\right)$, we see that $S_{2 n}-S_{2 n-1}=o(1)$, and therefore that

$$
\lim _{n \rightarrow \infty} S_{n}=\sum_{k=1}^{\infty} \frac{(-1)^{k} r_{2}(k)}{k}=-\pi \log 2 .
$$

This shows that the method of expanding circles yields $-\pi \log 2$ as the value of the lattice sum

$$
\sum_{i^{2}+j^{2}>0} \frac{(-1)^{i+j}}{i^{2}+j^{2}}
$$


We show next that the method of expanding diamonds yields the same value. This amounts to proving that

$$
T_{n}:=\sum_{0<|i|+|j| \leq n} \frac{(-1)^{i+j}}{i^{2}+j^{2}} \rightarrow-\pi \log 2 \text { as } n \rightarrow \infty .
$$

Observe that, for $0<t<1$,

$$
f(t):=\sum_{k=1}^{\infty} r_{2}(k) t^{k-1}=\frac{1}{t}\left(2 \sum_{i=0}^{\infty} t^{i^{2}}-1\right)^{2}-\frac{1}{t}=\frac{4}{t}\left(\sum_{i=0}^{\infty} t^{i^{2}}\right)^{2}-\frac{4}{t} \sum_{i=0}^{\infty} t^{i^{2}},
$$

and hence, by what has been proved above, that

$$
\int_{0}^{1} f(-t) d t=\pi \log 2 .
$$

But we also have

$$
\begin{aligned}
-f(-t) & =4 \sum_{k=0}^{\infty}(-1)^{k}\left(\sum_{j=0}^{k} t^{(k-j)^{2}+j^{2}-1}-t^{k^{2}-1}\right) \\
& =4 \sum_{k=1}^{\infty}(-1)^{k} \sum_{j=0}^{k-1} t^{(k-j)^{2}+j^{2}-1},
\end{aligned}
$$

so that

$$
-\int_{0}^{1} f(-t) d t=4 \sum_{k=1}^{\infty}(-1)^{k} \sum_{j=0}^{k-1} \frac{1}{(k-j)^{2}+j^{2}}=\lim _{n \rightarrow \infty} T_{n},
$$

provided we can justify the term-by-term integration. This can be done as follows: Note that, for $0<t<1$,

$$
-\int_{0}^{t} f(-u) d u=4 \sum_{k=1}^{\infty}(-1)^{k} \delta_{k}(t),
$$

where

$$
\delta_{k}(t):=\sum_{j=0}^{k-1} \frac{t^{(k-j)^{2}+j^{2}-1}}{(k-j)^{2}+j^{2}}
$$

and that, for $0 \leq j \leq k$,

$$
\frac{k^{2}}{2} \leq(k-j)^{2}+j^{2} \leq k^{2} .
$$

Further, for $0<t<1, k \geq 2$,

$$
\begin{aligned}
\delta_{k-1}(t)-\delta_{k}(t)= & \sum_{j=1}^{k-1}\left(\frac{t^{(k-j)^{2}+j^{2}-1}}{(k-j)^{2}+(j-1)^{2}}-\frac{t^{(k-j)^{2}+j^{2}-1}}{(k-j)^{2}+j^{2}}\right) \\
& +\sum_{j=1}^{k-1} \frac{t^{(k-j)^{2}-1}\left[t^{(j-1)^{2}}-t^{j^{2}}\right]}{(k-j)^{2}+(j-1)^{2}}-\frac{t^{k^{2}-1}}{k^{2}}
\end{aligned}
$$


so that

$$
\begin{aligned}
\left|\delta_{k-1}(t)-\delta_{k}(t)\right| \leq & \sum_{j=1}^{k-1}\left(\frac{1}{(k-j)^{2}+(j-1)^{2}}-\frac{1}{(k-j)^{2}+j^{2}}\right) \\
& +\sum_{j=1}^{k-1} \frac{t^{(j-1)^{2}}-t^{j^{2}}}{(k-j)^{2}+(j-1)^{2}}+\frac{1}{k^{2}} \\
\leq \quad & \sum_{j=1}^{k-1} \frac{j^{2}-(j-1)^{2}}{(k-j)^{2}+(j-1)^{2}} \cdot \frac{1}{(k-j)^{2}+j^{2}} \\
& +\frac{2}{k^{2}} \sum_{j=1}^{k-1}\left[t^{(j-1)^{2}}-t^{j^{2}}\right]+\frac{1}{k^{2}} \\
\leq \quad & \frac{4}{k^{2}(k-1)^{2}} \sum_{j=1}^{k-1}\left[j^{2}-(j-1)^{2}\right]+\frac{2}{k^{2}}+\frac{1}{k^{2}}=\frac{7}{k^{2}} .
\end{aligned}
$$

Also, for $0<t<1$,

$$
0 \leq \delta_{k}(t) \leq \sum_{j=0}^{k-1} \frac{1}{(k-j)^{2}+j^{2}} \leq \frac{2 k}{k^{2}}=\frac{2}{k} \rightarrow 0 \text { as } k \rightarrow \infty .
$$

It follows, by the Weierstrass M-test, that

$$
\begin{aligned}
4 \sum_{k=1}^{\infty}(-1)^{k} \delta_{k}(1) & =-\lim _{t \rightarrow 1-} 4 \sum_{k=1}^{\infty}\left[\delta_{2 k-1}(t)-\delta_{2 k}(t)\right] \\
& =-\lim _{t \rightarrow 1-} \int_{0}^{t} f(-u) d u=-\int_{0}^{1} f(-u) d u,
\end{aligned}
$$

and this completes the proof of the expanding diamonds case.

Finally we shall show that the method of expanding squares also yields the value $-\pi \log 2$ for the lattice sum. In fact we shall deal with the slightly more general method of expanding rectangles. Let

$$
R_{m, n}:=\sum_{\substack{|i|+|j|>0 \\|i| \leq n,|j| \leq m}} \frac{(-1)^{i+j}}{i^{2}+j^{2}}, \quad R_{m, n}(t):=\sum_{\substack{|i|+|j|>0 \\|i| \leq n,|j| \leq m}} t^{i^{2}+j^{2}-1} .
$$

We shall prove that $R_{m, n} \rightarrow-\pi \log 2$ when $\mu:=\min (m, n) \rightarrow \infty$. Observe that

$$
R_{m, n}=-\int_{0}^{1} R_{m, n}(-t) d t
$$

and that, for $0<t<1$,

$$
\begin{aligned}
f(t)-R_{m, n}(t) & =\frac{1}{t}\left(\sum_{|i|>n,|j|>m} t^{i^{2}+j^{2}}+\sum_{|i| \leq n,|j|>m} t^{i^{2}+j^{2}}+\sum_{|i|>n,|j| \leq m} t^{i^{2}+j^{2}}\right) \\
& =\frac{4}{t}\left(\sum_{i=n+1}^{\infty} t^{i^{2}} \sum_{j=m+1}^{\infty} t^{j^{2}}+\sum_{i=0}^{n} t^{i^{2}} \sum_{j=m+1}^{\infty} t^{j^{2}}+\sum_{i=n+1}^{\infty} t^{i^{2}} \sum_{j=0}^{m} t^{j^{2}}\right) .
\end{aligned}
$$


Observe also that, for $0<t<1$,

$$
\left|\sum_{i=n+1}^{\infty}(-t)^{i^{2}}\right|<t^{(n+1)^{2}} \text { and }\left|\sum_{i=0}^{n}(-t)^{i^{2}}\right|<1 .
$$

Hence, for $0<t<1$,

$$
\left|f(-t)-R_{m, n}(-t)\right|<4 t^{(n+1)^{2}+(m+1)^{2}-1}+4 t^{(m+1)^{2}-1}+4 t^{(n+1)^{2}-1}<12 t^{(\mu+1)^{2}-1},
$$

and so

$$
\left|R_{m, n}+\pi \log 2\right|=\left|\int_{0}^{1} f(-t) d t-\int_{0}^{1} R_{m, n}(-t) d t\right| \leq \frac{12}{(\mu+1)^{2}} \rightarrow 0 \text { as } \mu \rightarrow \infty .
$$

This completes the proof.

\section{The Proofs}

Proof of Theorem 1. We first note that for a positive definite quadratic form

$$
Q\left(x_{1}, \ldots, x_{k}\right)=\sum_{i=1}^{k} \sum_{j=1}^{k} \alpha_{i j} x_{i} x_{j} \in \mathbf{R}\left[x_{1}, \ldots, x_{k}\right]
$$

with $\alpha_{i j}=\alpha_{j i}$ we have

$$
Q\left(x_{1}, \ldots, x_{k}\right) \geq \lambda\left(x_{1}^{2}+\cdots+x_{k}^{2}\right)
$$

for some $\lambda=\lambda_{Q}>0$. Suppose in what follows that $\sigma>(k-1) / 2, \nu>0$, and the $j_{i}$ are integers. Let

$$
Q(0, \ldots, 0, s):=0, \quad Q\left(j_{1}, \ldots j_{k}, s\right):=Q\left(j_{1}, \ldots, j_{k}\right)^{-s} \text { when } j_{1}^{2}+\cdots+j_{k}^{2} \neq 0,
$$

and let

$$
\mu:=\sup _{\substack{\nu>0 \\\left(j_{1}, \ldots, j_{k}\right) \in \mathbf{Z}^{k}}}\left|w_{\nu}\left(j_{1}, \ldots, j_{k}\right)\right|<\infty
$$

Then we have

$$
\begin{aligned}
& A_{\nu}(s) \\
& =\sum_{j_{1}=-\infty}^{\infty} \cdots \sum_{j_{k}=-\infty}^{\infty} q\left(j_{1}, \ldots, j_{k}\right) \chi_{\nu}\left(j_{1}, \ldots, j_{k}\right) Q\left(j_{1}, \ldots, j_{k}, s\right) \\
& =\sum_{j_{1}=-\infty}^{\infty} \cdots \sum_{j_{k}=-\infty}^{\infty} w_{\nu}\left(j_{1}, \ldots, j_{k}\right)\left\{Q\left(j_{1}, \ldots, j_{k-1}, j_{k}, s\right)-Q\left(j_{1}, \ldots, j_{k-1}, j_{k}+1, s\right)\right\} \\
& =: \sum_{j_{1}=-\infty}^{\infty} \cdots \sum_{j_{k}=-\infty}^{\infty} a_{j_{1}, \ldots, j_{k}}(\nu, s) .
\end{aligned}
$$

Suppose next that $\sigma>\frac{(k-1)}{2}+\epsilon>\frac{(k-1)}{2}$. Observe that, for $0<u<v$,

$$
\left|\frac{1}{u^{s}}-\frac{1}{v^{s}}\right|=\left|s \int_{u}^{v} t^{-1-s} d t\right| \leq \frac{v-u}{u^{1+\sigma}}|s|,
$$


and hence that, when $\left(j_{1}^{2}+\cdots+j_{k}^{2}\right)\left(j_{1}^{2}+\cdots+\left(j_{k}+1\right)^{2}\right) \neq 0$,

$$
\begin{aligned}
\left|a_{j_{1}, \ldots, j_{k}}(\nu, s)\right| & \leq \frac{\left|Q\left(j_{1}, \ldots, j_{k}\right)-Q\left(j_{1}, \ldots, j_{k}+1\right)\right|}{\lambda^{1+\sigma}\left(j_{1}^{2}+\cdots+j_{k}^{2}\right)^{1+\sigma}}|s| \mu \\
& =\frac{\left|2 \sum_{i=1}^{k-1} \alpha_{i k} j_{i}+\alpha_{k k}\left(2 j_{k}+1\right)\right|}{\lambda^{1+\sigma}\left(j_{1}^{2}+\cdots+j_{k}^{2}\right)^{1 / 2}} \frac{|s| \mu}{\left(j_{1}^{2}+\cdots+j_{k}^{2}\right)^{\sigma+1 / 2}} \\
& \leq \frac{\lambda^{-1-\sigma}|s| M}{\left(j_{1}^{2}+\cdots+j_{k}^{2}\right)^{\sigma+1 / 2}},
\end{aligned}
$$

where

$$
M:=\sup _{j_{1}^{2}+\cdots+j_{k}^{2}>0} \frac{\left|2 \sum_{i=1}^{k-1} \alpha_{i k} j_{i}+\alpha_{k k}\left(2 j_{k}+1\right)\right|}{\left(j_{1}^{2}+\cdots+j_{k}^{2}\right)^{1 / 2}} \mu<\infty .
$$

Also, when $\left(j_{1}^{2}+\cdots+j_{k}^{2}\right)\left(j_{1}^{2}+\cdots+\left(j_{k}+1\right)^{2}\right)=0$,

$$
\left|a_{j_{1}, \ldots, j_{k}}(\nu, s)\right| \leq \frac{\mu}{\left|\alpha_{k, k}\right|^{\sigma}} .
$$

Since (as in Borwein-Borwein-Taylor [1])

$$
\sum_{j_{1}^{2}+\cdots+j_{k}^{2}>0} \frac{1}{\left(j_{1}^{2}+\cdots+j_{k}^{2}\right)^{(k / 2)+\epsilon}}<\infty
$$

it follows, by the Weierstrass M-test, that when $\nu \rightarrow \infty, A_{\nu}(s) \rightarrow A(s)$ say, uniformly in the region $\{s: \sigma>(k-1) / 2+\epsilon,|s| \leq K\}$ with $K$ any fixed positive number, and, since $A_{\nu}(s)$ is analytic in this region, that $A(s)$ is analytic therein. Consequently

$$
A(s)=\lim _{n \rightarrow \infty} A_{n}(s)
$$

exists and is analytic in the region $\operatorname{Re} s>(k-1) / 2$.

Proof of Theorem 2. We suppose that $C$ is the diamond $a_{1}\left|x_{1}\right|+\cdots+a_{k}\left|x_{k}\right| \leq c$ where $d=\operatorname{gcd}\left(a_{1}, \ldots, a_{k}\right)$ and all the $a_{i} / d$ are odd positive integers. Observing that when $a_{1}\left|x_{1}\right|+\cdots+a_{k}\left|x_{k}\right|=c n$ and $n$ is a multiple of $d$ we have

$$
(-1)^{x_{1}+\cdots+x_{k}}=(-1)^{c n / d}
$$

and

$$
\left|Q\left(x_{1}, \cdots, x_{k}\right)\right| \leq\left(\sum_{i=1}^{k} \sum_{j=1}^{k}\left|\alpha_{i j}\right|\right) c^{2} n^{2},
$$

it is not hard to see that when $s=\frac{1}{2}(k-1)$ and $N$ is a multiple of $B:=a_{1} \cdots a_{k}$

$$
\begin{aligned}
\left|A_{N}(s)-A_{N-1 / c}(s)\right| & =\sum_{a_{1}\left|x_{1}\right|+\cdots+a_{k}\left|x_{k}\right|=c N} Q\left(x_{1}, \ldots, x_{k}\right)^{-\frac{1}{2}(k-1)} \\
& >\frac{1}{c_{1} N^{k-1}} \sum_{\left|x_{1}^{\prime}\right|+\cdots+\left|x_{k}^{\prime}\right|=(c N / B)} 1 \\
& \geq \frac{1}{c_{1} N^{k-1}}\left(\begin{array}{c}
(c N / B)+k-1 \\
k-1
\end{array}\right) \\
& >c_{2}>0
\end{aligned}
$$

as $N \rightarrow \infty$ (where in fact we have only bothered to count the points with $x_{i}=$ $\left.\left(B / a_{i}\right) x_{i}^{\prime} \geq 0\right)$; and the limit cannot exist. 
Proof of Corollary 1. Under the hypothesis, it is simple to estimate that

$$
w_{\nu}\left(j_{1}, \ldots, j_{k-1}, m\right):=\sum_{l=-\infty}^{m} q\left(j_{1}, \ldots, j_{k-1}, l\right) \chi_{\nu}\left(j_{1}, \ldots, j_{k-1}, l\right)
$$

is uniformly bounded.

Proof of Theorem 3. Suppose that $q\left(x_{1}, \ldots, x_{k}\right)$ is periodic with period $M$. Then, (with $S_{0}(x)$ as defined in (1)) dividing the sum into residue classes modulo $M$, we have

$$
S_{0}(x)=\sum_{r_{1}=1}^{M} \cdots \sum_{r_{k}=1}^{M} q\left(r_{1}, \ldots, r_{k}\right) F_{0}\left(r_{1}, \ldots, r_{k}, x\right)
$$

where

$$
F_{0}\left(r_{1}, \ldots, r_{k}, x\right):=\sum_{\substack{Q\left(x_{1}, \ldots, x_{k}\right) \leq x \\ x_{i} \equiv r_{i} \bmod M}} 1 .
$$

Writing $x_{i}=M y_{i}+r_{i}$, it is easy to see that $F\left(r_{1}, \ldots, r_{k}, x\right)$ counts the number of lattice points $\left(y_{1}, \ldots, y_{k}\right) \in \mathbf{Z}^{k}$ in the expanded ellipse $x^{1 / 2} E$, where $E$ is the ellipse $M^{2} Q\left(y_{1}, \ldots, y_{k}\right) \leq 1$ of area

$$
A=\frac{\pi^{k / 2}}{M^{k} \sqrt{|D|} \Gamma(k / 2+1)}
$$

with centre shifted to $\left(-r_{1} / M, \ldots,-r_{k} / M\right)$. Approximating the number of points in the ellipse by its area, we can write

$$
F_{0}\left(r_{1}, \ldots, r_{k}, x\right)=A x^{k / 2}+O(\psi),
$$

where from the results of Huxley [7, Theorem 5] (for $k=2$ ), Krätzel \& Nowak [9] (for $\mathrm{k}=3$ ), and Walfisz [17] and Landau [10,11] (for $k \geq 8$ and $k \geq 4$ respectively; see Landau [12, Satz II] for the most immediately applicable form) we can take

$$
\psi= \begin{cases}x^{23 / 73}(\log x)^{315 / 146} & \text { when } k=2, \\ x^{25 / 34}(\log x)^{10 / 17} & \text { when } k=3 \\ x \log ^{2} x & \text { when } k=4 \\ x^{(k / 2)-1} & \text { when } k \geq 5 .\end{cases}
$$

Hence

$$
S_{0}(x)=B_{1} x^{k / 2}+O\left(B_{2} \psi\right)
$$

where

$$
B_{1}:=\sum_{r_{1}=1}^{M} \ldots \sum_{r_{k}=1}^{M} q\left(r_{1}, \ldots, r_{k}\right), \quad B_{2}:=\sum_{r_{1}=1}^{M} \cdots \sum_{r_{k}=1}^{M}\left|q\left(r_{1}, \ldots, r_{k}\right)\right|,
$$

and the result is plain from (2).

Proof of Theorem 4. Notice that if $Q\left(x_{1}, \ldots, x_{k}\right)=a_{1} x_{1}^{2}+\cdots+a_{k} x_{k}^{2}$ with all the $a_{i}$ odd positive integers and $q\left(x_{1}, \ldots, x_{k}\right)=(-1)^{x_{1}+\cdots+x_{k}}$, then

$$
r(n, Q, q)=(-1)^{n} \sum_{Q\left(x_{1}, \ldots, x_{k}\right)=n} 1 .
$$

Now by elementary methods we have

$$
\sum_{n \leq x}|r(n, Q, q)|=\sum_{Q\left(x_{1}, \ldots, x_{k}\right) \leq x} 1=A(1+o(1)) x^{k / 2}
$$


as $x \rightarrow \infty$, where $A$ is the area of the ellipse $Q\left(x_{1}, \ldots, x_{k}\right) \leq 1$. In particular there must certainly be infinitely many integers $n$ with $|r(n, Q, q)|>\frac{1}{2} A n^{(k / 2)-1}$. Hence if $\operatorname{Re} s \leq(k / 2)-1$ then

$$
\left|A_{N}(s)-A_{N-1}(s)\right|=\frac{|r(N, Q, q)|}{N^{\sigma}} \not \supset 0
$$

as $N \rightarrow \infty$, and the limit $A(s)$ cannot exist.

Proof of Theorem 5. We closely follow the proof of the corresponding omega result for the error in the classical circle problem (as given in Landau [13]) and for $\rho \geq 1$ inductively define

$$
S_{\rho+1}(x):=\int_{0}^{x} S_{\rho}(u) d u
$$

where $S_{0}(x)$ is as defined in (1) (so that equivalently

$$
S_{\rho}(x)=\frac{1}{\rho !} \sum_{0 \leq n \leq x}(x-n)^{\rho} r(n, Q, q)=\frac{1}{(\rho-1) !} \int_{0}^{x} S_{0}(u)(x-u)^{\rho-1} d u
$$

for all $\rho \geq 1)$.

We invoke the following lemma of M. Riesz [16] (as in Wilton [20]; cf. Landau [13, Satz 533]):

Lemma 1. If $f_{0}(x)$ is L-integrable and bounded over $(0, x)$ and if, when $\gamma>0$ and $x>0$,

$$
f_{\gamma}(x):=\frac{1}{\Gamma(\gamma)} \int_{0}^{x} f_{0}(u)(x-u)^{\gamma-1} d u
$$

and if, further, $V(x)$ and $W(x)$ are increasing functions of $x$ with

$$
\left|f_{0}(x)\right|<V(x)
$$

and

$$
\left|f_{l}(x)\right|<W(x)
$$

then

$$
\left|f_{\beta}(x)\right|<b(\beta, l) V(x)^{(1-(\beta / l))} W(x)^{(\beta / l)}
$$

for all $0 \leq \beta \leq l$, where the $b(\beta, l)$ depend only on $\beta$ and $l$.

We shall show that (as long as $A_{\nu}(s)$ is not identically zero) there are a positive integer $\nu$ and non-zero constants $B_{\nu+1}$ and $C_{\nu}$ such that

$$
\left|S_{\nu+1}(x)\right|<B_{\nu+1}(1+o(1)) x^{\frac{1}{4}(k+2(\nu+1)-1)}
$$

for all $x$ and

$$
\left|S_{\nu}(x)\right|>\left|C_{\nu}\right|(1+o(1)) x^{\frac{1}{4}(k+2 \nu-1)}
$$

for infinitely many $x$.

Hence by the above lemma we must have

$$
\left|S_{0}(x)\right| \geq(1+o(1))\left(\frac{\left|C_{\nu}\right|}{b(\nu, \nu+1) B_{\nu+1}^{\nu /(\nu+1)}}\right)^{\nu+1} x^{\frac{1}{4}(k-1)}
$$

for infinitely many $x$. 
Thus it remains to justify the claimed upper and lower bounds for the $S_{\rho}(x)$. We define the constants

$$
\begin{aligned}
C_{\rho} & :=\frac{(\sqrt{D} M)^{\frac{1}{2}(k+2 \rho-1)}}{M \pi^{\rho+1}}\left(\sum_{n=1}^{\infty} \frac{r^{*}(n)}{n^{\frac{1}{4}(k+2 \rho+1)}}\right) \cos \left((k+2 \rho+1) \frac{\pi}{4}\right), \\
B_{\rho} & :=\frac{(\sqrt{D} M)^{\frac{1}{2}(k+2 \rho-1)}}{M \pi^{\rho+1}}\left(\sum_{n=1}^{\infty} \frac{\left|r^{*}(n)\right|}{n^{\frac{1}{4}(k+2 \rho+1)}}\right) .
\end{aligned}
$$

Lemma 2. We suppose that $q\left(x_{1}, \ldots, x_{k}\right)$ is periodic in all the $x_{i}$ with period $M$ and

$$
\sum_{r_{1}=1}^{M} \cdots \sum_{r_{k}=1}^{M} q\left(r_{1}, \ldots, r_{k}\right)=0 .
$$

For all $\rho>\frac{1}{2}(k-1)$ we have

$$
\left|S_{\rho}(x)\right| \leq B_{\rho}\left(1+O\left(x^{-1 / 2}\right)\right) x^{\frac{1}{4}(k+2 \rho-1)}
$$

for all $x$, and if $C_{\rho} \neq 0$

$$
\left|S_{\rho}(x)\right|=C_{\rho}\left(1+O\left(\left(\frac{\log x}{\log \log x}\right)^{-1 / 4}\right)\right) x^{\frac{1}{4}(k+2 \rho-1)}
$$

for infinitely many integers $x$.

Proof. For $\rho \geq 0$ we inductively define

$$
F_{\rho+1}(\vec{r}, x):=\int_{0}^{x} F_{\rho}(\vec{r}, u) d u,
$$

and observe (by repeated integration of (6)) that

$$
S_{\rho}(x)=\sum_{r_{1}=1}^{M} \cdots \sum_{r_{k}=1}^{M} q\left(r_{1}, \ldots, r_{k}\right) F_{\rho}\left(r_{1}, \ldots, r_{k}, x\right) .
$$

It has been shown by a number of authors (see for example Landau [10]) that the $F_{\rho}(\vec{r}, x)$ can be expressed in terms of Bessel functions:

$$
F_{\rho}(\vec{r}, x)=V_{\rho}(x)+\frac{(\sqrt{D} M)^{\frac{1}{2}(k+2 \rho-2)}}{M \pi^{\rho}} E(\vec{r}, x),
$$

where

$$
V_{\rho}(x):=\frac{\pi^{k / 2}}{M^{k} D \Gamma(k / 2+\rho+1)} x^{k / 2+\rho}
$$

and

$$
E(\vec{r}, x):=x^{k / 4+\rho / 2} \sum_{n=1}^{\infty} \frac{r^{*}(n ; \vec{r})}{n^{k / 4+\rho / 2}} J_{k / 2+\rho}\left(\frac{2 \pi}{M} \sqrt{\frac{n x}{D}}\right),
$$

with

$$
r^{*}(n ; \vec{r}):=\sum_{\substack{\vec{x} \in \mathbf{Z}^{k} \\ Q^{*}(\vec{x})=n}} \cos \left(\frac{2 \pi}{M} \vec{r} \cdot \vec{x}\right) .
$$

Notice that, from the straightforward bounds

$$
\sum_{\substack{\vec{x} \in \mathbf{Z}^{k} \\ Q^{*}(\vec{x}) \leq z}} 1=O\left(z^{k / 2}\right), \quad J_{\nu}(z)=O\left(z^{-1 / 2}\right),
$$


such a sequence is absolutely convergent for $\rho>(k-1) / 2$.

Hence if

$$
\sum_{r_{1}=1}^{M} \cdots \sum_{r_{k}=1}^{M} q\left(r_{1}, \ldots, r_{k}\right)=0
$$

we obtain

$$
S_{\rho}(x)=\frac{(\sqrt{D} M)^{\frac{1}{2}(k+2 \rho-2)}}{M \pi^{\rho}} x^{k / 4+\rho / 2} \sum_{n=1}^{\infty} \frac{r^{*}(n)}{n^{k / 4+\rho / 2}} J_{k / 2+\rho}\left(\frac{2 \pi}{M} \sqrt{\frac{n x}{D}}\right) .
$$

Approximating the Bessel functions by cosines (for example see Watson [19, p. 199]);

$$
J_{\nu}(z)=\sqrt{\frac{2}{\pi z}} \cos \left(z-(2 \nu+1) \frac{\pi}{4}\right)+O\left(z^{-3 / 2}\right)
$$

we obtain

$$
S_{\rho}=\frac{(\sqrt{D} M)^{\frac{1}{2}(k+2 \rho-1)}}{M \pi^{\rho+1}} x^{(k+2 \rho-1) / 4}\left(M_{1}+M_{2}\right)
$$

with

$$
M_{1}:=\sum_{n=1}^{\infty} \frac{r^{*}(n)}{n^{(k+2 \rho+1) / 4}} \cos \left(\frac{2 \pi}{M} \sqrt{\frac{n x}{D}}-(k+2 \rho+1) \frac{\pi}{4}\right)
$$

and

$$
M_{2}:=O\left(x^{-1 / 2} \sum_{n=1}^{\infty} \frac{r^{*}(n)}{n^{(k+2 \rho+3) / 4}}\right)=O\left(x^{-1 / 2}\right)
$$

(the latter bound since

$$
\sum_{n \leq z}\left|r^{*}(n)\right|=O\left(z^{k / 2}\right)
$$

and by assumption $2 \rho+1>k$ ).

The trivial bound

$$
\left|M_{1}\right| \leq \sum_{n=1}^{\infty} \frac{\left|r^{*}(n)\right|}{n^{(k+2 \rho+1) / 4}}
$$

gives us the required upper bound.

By the box principle (in $k$ dimensions), given an $N$ and $k$ real numbers $\nu_{1}, \ldots$, $\nu_{k}$, there is certainly an integer $1 \leq m \leq\left(N^{k}+1\right)$ such that the distances from the $m \nu_{i}$ to their nearest integers simultaneously satisfy $\left\|m \nu_{i}\right\|<1 / N$. In particular (taking $k=N)$ there is an integer $m=\sqrt{z} \leq N^{N}+1$ with $m(\sqrt{n} / M \sqrt{D})$ close enough to an integer for $n=1, \ldots, N$ that

$$
\begin{aligned}
\cos \left(\frac{2 \pi}{M} \sqrt{\frac{n z}{|D|}}-(k+2 \rho+1) \frac{\pi}{4}\right) & =\cos \left((k+2 \rho+1) \frac{\pi}{4}\right)+O\left(\frac{1}{N}\right) \\
& =\cos \left((k+2 \rho+1) \frac{\pi}{4}\right)+O\left(\frac{\log \log 16 z}{\log 16 z}\right)
\end{aligned}
$$

for all $n=1, \ldots, N$ (the latter equality following from the observation that, since $\log 16 z>e$,

$$
\left.\frac{(\log 16 z)}{\log (\log 16 z)} \leq \frac{\log \left(16\left(N^{N}+1\right)^{2}\right)}{\log \log \left(16\left(N^{N}+1\right)^{2}\right)}=O(N)\right)
$$


Using the estimate (7), we can readily bound the remaining terms in the sum by

$$
\begin{aligned}
\sum_{n=N}^{\infty} \frac{r^{*}(n)}{n^{(k+2 \rho+1) / 4}} & =O\left(N^{-(2 \rho+1-k) / 4}\right) \\
& =O\left(N^{-1 / 4}\right)=O\left(\left(\frac{\log \log 16 z}{\log 16 z}\right)^{1 / 4}\right)
\end{aligned}
$$

Hence for such a $z$, as long as $C_{\rho} \neq 0$, we have

$$
M_{1}=\cos \left((k+2 \rho+1) \frac{\pi}{4}\right)\left(1+O\left(\left(\frac{\log \log 16 z}{\log 16 z}\right)^{1 / 4}\right)\right)\left(\sum_{n=1}^{\infty} \frac{r^{*}(n)}{n^{(k+2 \rho+1) / 4}}\right) \text {, }
$$

and the remaining bound is plain. Varying $N$ (and hence the closeness of the approximation), we can clearly generate infinitely many integers $z$ in this way.

Final step of the proof. Hence it only remains to justify that (as long as $r(n, Q, q)$ is not identically zero) $C_{\rho}$ is non-zero for some $\rho$.

First observe that $r^{*}(n)$ cannot be identically zero: If $r^{*}(n)$ is identically zero then $S_{\rho}(x)=0$ for all $x \geq 0$ and any $\rho>(k-1) / 2$; in particular it follows from the relation

$$
r(N, Q, q)=(N+1) ! S_{\rho}(N+1)-\sum_{n=0}^{N-1}(N+1-n)^{\rho} r(n, Q, q)
$$

and an easy induction on $N$ that $r(n, Q, q)$ must be identically zero.

We suppose that $w$ is the smallest positive integer such that $r^{*}(w) \neq 0$. From the lower bound $Q^{*}\left(x_{1}, \ldots, x_{k}\right)>\lambda^{*}\left(x_{1}^{2}+\cdots+x_{k}^{2}\right)$ we certainly have the trivial lower bound

$$
\left|r^{*}(n)\right| \leq B n^{k / 2}, \quad B:=\left(\sum_{r_{1}=1}^{M} \cdots \sum_{r_{k}=1}^{M}\left|q\left(r_{1}, \ldots, r_{k}\right)\right|\right)\left(\frac{2}{\sqrt{\lambda^{*}}}\right)^{k} .
$$

Hence if

$$
R \geq N:=\max \left\{\frac{k}{2}+2, \log \left(\frac{\pi^{2}}{12} \frac{B}{\left|r^{*}(w)\right|}(w+1)^{k / 2+2}\right) / \log \left(1+\frac{1}{w}\right)\right\},
$$

we have

$$
\left|\sum_{n=w+1}^{\infty} \frac{r^{*}(n)}{n^{R}}\right|<\frac{B(w+1)^{k / 2+2}}{(w+1)^{R}}\left(\sum_{n=1}^{\infty} \frac{1}{n^{2}}\right) \leq\left|\frac{r^{*}(w)}{w^{R}}\right|,
$$

and $\sum_{n=1}^{\infty} r^{*}(n) / n^{R} \neq 0$. In particular at least one of $\rho=[(4 N-1-k) / 2]+1$ or $\rho=$ $[(4 N-1-k) / 2]+1$ will have both $\sum_{n=1}^{\infty} r^{*}(n) / n^{(k+2 \rho+1) / 4}$ and $\cos ((k+2 \rho+1) \pi / 4)$ non-zero, and hence $C_{\rho} \neq 0$. 
Proof of the representation (4). By partial summation and integration by parts we obtain

$$
\begin{aligned}
& A_{\nu}(s)=\frac{S_{0}(\nu)-q(\overrightarrow{0})}{\nu^{s}}-\int_{1}^{\nu}\left(S_{0}(u)-q(\overrightarrow{0})\right) \frac{d}{d u}\left(u^{-s}\right) d u \\
& =\sum_{i=0}^{\rho} \frac{\Gamma(s+i)}{\Gamma(s)}\left(\frac{S_{i}(\nu)}{\nu^{s+i}}-S_{i}(1)\right)+\frac{\Gamma(s+\rho+1)}{\Gamma(s)} \int_{1}^{\nu} S_{\rho}(u) u^{-s-\rho-1} d u \\
& =\sum_{i=0}^{\rho-1} \frac{\Gamma(s+i)}{\Gamma(s)}\left(\frac{S_{i}(\nu)}{\nu^{s+i}}-S_{i}(1)\right)-\frac{\Gamma(s+\rho)}{\Gamma(s)} S_{\rho}(1) \\
& \quad+\frac{\Gamma(s+\rho+1)}{\Gamma(s)} \int_{1}^{\infty} S_{\rho}(u) u^{-s-\rho-1} d u+O\left(\nu^{-\frac{1}{2}\left(\rho-\frac{1}{2}(k-1)+2 \sigma\right)}\right),
\end{aligned}
$$

for bounded $|s|$, since by Lemma 2

$$
S_{\rho}(u)=O\left(u^{\frac{1}{4}(k+2 \rho-1)}\right)
$$

for $\rho>\frac{1}{2}(k-1)$.

Proof of Theorem 6. Writing

$$
r(n, \vec{r})=r\left(n ; r_{1}, \ldots, r_{k}\right):=\sum_{\substack{Q\left(x_{1}, \ldots, x_{k}\right)=n \\ x_{i} \equiv r_{i}(\bmod M)}} 1,
$$

for Re $s>0$ we define the $\theta$-function

$$
\begin{aligned}
\hat{\theta}(s) & :=\sum_{n=0}^{\infty} r(n, Q, q) e^{-n s} \\
& =\sum_{r_{1}=1}^{M} \cdots \sum_{r_{k}=1}^{M} q\left(r_{1}, \ldots, r_{k}\right) \hat{\theta}(s ; \vec{r})
\end{aligned}
$$

where

$$
\hat{\theta}(s ; \vec{r}):=\sum_{n=0}^{\infty} r(n ; \vec{r}) e^{-n s} .
$$

Now by Novák [14, Lemma 1] for integers $h$ and $l$ (with $(h, l)=1$ and $l>0$ ) the modular functions $\hat{\theta}(s ; \vec{r})$ can be expanded in a neighbourhood of the cusp $2 \pi i h / l$ so that for $\operatorname{Re} s>0$

$$
\hat{\theta}(s ; \vec{r})=\frac{\pi^{k / 2}\left(s-\frac{2 \pi i h}{l}\right)^{-k / 2}}{\sqrt{D} M^{k / 2} l^{k}} \sum_{\vec{m} \in \mathbf{Z}^{k}} S_{h, l}(\vec{m}, \vec{r}) \exp \left(-\frac{\pi^{2} Q^{*}(\vec{m})}{D M^{2} l^{2}\left(s-\frac{2 \pi i h}{l}\right)}\right),
$$

where

$$
S_{h, l}(\vec{m}, \vec{r}):=\sum_{a_{1}=1}^{l} \cdots \sum_{a_{k}=1}^{l} \exp \left(-\frac{2 \pi i h}{l} Q(\vec{a} M+\vec{r})+\frac{2 \pi i}{l M} \vec{m} \cdot(\vec{a} M+\vec{r})\right) .
$$

Thus

$$
\hat{\theta}\left(\sigma+\frac{2 \pi i h}{l}, \vec{r}\right)=\frac{\pi^{k / 2}}{\sqrt{D} M^{k / 2} l^{k} \sigma^{k / 2}}\left(S_{h, l}(\overrightarrow{0}, \vec{r})+E_{h, l}(\vec{r}, \sigma)\right)
$$


where for fixed $h$ and $l$

$$
\begin{aligned}
E_{h, l}(\vec{r}, \sigma) \leq & \sum_{\substack{\vec{m} \in \mathbf{Z}^{k} \\
\vec{m} \neq \overrightarrow{0}}} l^{k} \exp \left(-\frac{\pi^{2} \lambda^{*}}{D M^{2} l^{2} \sigma}\left(m_{1}^{2}+\cdots+m_{k}^{2}\right)\right) \\
& =O\left(\sum_{n=1}^{\infty} n^{k / 2} \exp (-c n / \sigma)\right)=O\left(e^{-c / \sigma}\right) .
\end{aligned}
$$

Now if we are in the non-singular case we can pick $h$ and $l$ such that

$$
A:=\frac{\pi^{k / 2}}{\sqrt{D} M^{k / 2} l^{k}} \sum_{r_{1}=1}^{M} \cdots \sum_{r_{k}=1}^{M} q\left(r_{1}, \ldots, r_{k}\right) S_{h, l}(\overrightarrow{0}, \vec{r}) \neq 0
$$

(notice that, since the $q\left(r_{1}, \ldots, r_{k}\right)$ sum to zero, $h \neq 0$ ) and hence

$$
\sigma^{k / 2} \theta\left(\sigma+\frac{2 \pi i h}{l}\right)=A+O\left(e^{-c / \sigma}\right)
$$

uniformly in $\sigma$.

Now, with $S_{0}(x)$ as in (1), writing

$$
\hat{\theta}(s)=s \int_{0}^{\infty} e^{-x s} S_{0}(x) d x,
$$

it is clear that $\left|S_{0}(x)\right|<c x^{k / 2-1}$ for all $x$ would imply that

$$
\begin{aligned}
\sigma^{k / 2} \hat{\theta}\left(\sigma+\frac{2 \pi i h}{l}\right) & <c|\sigma+(2 \pi i h / l)| \sigma^{k / 2} \int_{0}^{\infty} e^{-\sigma x} x^{k / 2-1} d x \\
& =c|\sigma+(2 \pi i h / l)| \Gamma(k / 2) .
\end{aligned}
$$

Hence, on letting $\sigma \rightarrow 0$, we see that for any constant

$$
c_{1}<A(2 \pi h / l)^{-1}(\Gamma(k / 2))^{-1}
$$

we must have $\left|S_{0}(x)\right|>c_{1} x^{k / 2-1}$ for infinitely many integers $x$.

Proof of Corollary 2. We take $h=1$ and $l$ to be a high power of $M$,

$$
l:=M^{\gamma}, \quad \gamma>2 \alpha,
$$

where $\alpha$ is the highest power of a prime factor of $M$ dividing $2^{k} D$ (recall that $D$, defined in (3), is the determinant of the matrix of coefficients $\alpha_{i j}$ of $Q\left(x_{1}, \ldots, x_{k}\right)$ ).

Writing

$$
\mathcal{R}:=\left\{\left(x_{1}, \ldots, x_{k}\right) \in \mathbf{Z}^{k}: 1 \leq x_{i} \leq l\right\}
$$

and

$$
F(\vec{x}):=\exp \left(-\frac{2 \pi i}{l} Q(\vec{x})\right) q(\vec{x}),
$$

non-singularity will follow once we show the non-vanishing of

$$
S:=M^{-k} \sum_{x_{1}=1}^{l M} \cdots \sum_{x_{k}=1}^{l M} F(\vec{x})=\sum_{\vec{x} \in \mathcal{R}} F(\vec{x}) .
$$


Now

$$
\begin{aligned}
|S|^{2} & =\sum_{\vec{x} \in \mathcal{R}} \sum_{\vec{y} \in \mathcal{R}} F(\vec{y}) \overline{F(\vec{x})} \\
& =\sum_{\vec{x} \in \mathcal{R}} \sum_{\vec{u} \in \mathcal{R}} F(\vec{u}+\vec{x}) \overline{F(\vec{x})} \\
& =\sum_{\vec{u} \in \mathcal{R}} F(\vec{u}) \prod_{i=1}^{k}\left(\sum_{x_{i}=1}^{l} \exp \left(-\frac{2 \pi i}{l} x_{i}\left(2 \sum_{j=1}^{k} \alpha_{i j} u_{j}\right)\right),\right.
\end{aligned}
$$

on noting that

$$
q(\vec{u}+\vec{x})=q(\vec{u}) q(\vec{x})
$$

and expanding

$$
Q(\vec{u}+\vec{x})-Q(\vec{x})=Q(\vec{u})+\sum_{i=1}^{k} x_{i}\left(2 \sum_{j=1}^{k} \alpha_{i j} u_{j}\right) .
$$

Observing that

$$
\sum_{x_{i}=1}^{l} \exp \left(-\frac{2 \pi i x_{i} \alpha}{l}\right)= \begin{cases}l & \text { if } \alpha \equiv 0(\bmod l) \\ 0 & \text { otherwise }\end{cases}
$$

and setting

$$
L:=\left\{\left(u_{1}, \ldots, u_{k}\right) \in \mathcal{R}: 2 \sum_{j=1}^{k} \alpha_{i j} u_{j} \equiv 0(\bmod l), 1 \leq i \leq k\right\},
$$

we obtain

$$
|S|^{2}=l^{k} \sum_{\vec{u} \in L} F(\vec{u})
$$

Now it is not hard to check that any $\vec{u}$ satisfying the linear system in $L$ must necessarily satisfy

$$
2^{k} D u_{i} \equiv 0(\bmod l), \quad 1 \leq i \leq k,
$$

and therefore certainly

$$
u_{i} \equiv 0\left(\bmod M^{\gamma-\alpha}\right), \quad 1 \leq i \leq k .
$$

Since we have chosen $\gamma>2 \alpha$, we thus have

$$
q(\vec{u})=1, \quad Q(\vec{u}) \equiv 0(\bmod l),
$$

for any $\vec{u} \in L$, giving

$$
|S|^{2}=\sum_{\vec{u} \in L} 1 \neq 0
$$

(plainly $(l, \ldots, l)$ is in $L$ ), and we are in the non-singular situation for any $Q\left(x_{1}, \ldots, x_{k}\right)$.

Proof of Proposition 1. Clearly it is enough to show the result for triangles T, and in fact (by taking sums and differences) enough to consider triangles with one vertex at the origin. Using symmetry $x \rightarrow-x, y \rightarrow-y$, we shall further assume that the triangle $T$ lies entirely in the quadrant $x, y \geq 0$ and (replacing $N$ by $c N$ or $N / \operatorname{gcd}(a, b)$ as necessary) that $T$ takes the form

$$
T=\left\{(x, y): r_{2} x \geq s_{2} y, r_{1} x \leq s_{1} y, a x-b y \leq 1\right\},
$$


where $a, b, s_{i}, r_{i} \in Z$ with $r_{i}, s_{i} \geq 0$ and $\operatorname{gcd}(a, b)=\operatorname{gcd}\left(r_{i}, s_{i}\right)=1$. We denote the sides of $T$ by $l_{i}$ :

$$
l_{1}: r_{1} x=s_{1} y, \quad l_{2}: r_{2} x=s_{2} y, l_{3}: a x-b y=1,
$$

and by $P_{1}, P_{2}$ the points of intersection of $l_{3}$ with $l_{1}$ and $l_{2}$ respectively;

$$
P_{i}=\left(\frac{s_{i}}{a s_{i}-b r_{i}}, \frac{r_{i}}{a s_{i}-b r_{i}}\right)
$$

Choosing integers $x_{0}, y_{0}$ satisfying

$$
a x_{0}-b y_{0}=1
$$

and writing

$$
A_{i}=r_{i} x_{0}-s_{i} y_{0}, \quad B_{i}=a s_{i}-b r_{i}, \quad \alpha_{i}=\frac{A_{i}}{B_{i}}
$$

(notice that $\operatorname{gcd}\left(A_{i}, B_{i}\right)=1$ and $B_{i}>0$ ), we can parametrise the integer points on $n\left(l_{3} \cap T\right)$ (the intersection of the line $a x-b y=n$ with $N T$ ) by

$$
x=n x_{0}+b t, y=n y_{0}+a t, n \alpha_{1} \leq t \leq n \alpha_{2}
$$

for $n=1, \ldots, N$, with $(-1)^{x+y}=(-1)^{n\left(x_{0}+y_{0}\right)+t(a+b)}$. We distinguish two cases:

(i) $2 \mid a b$

(ii) $2 \nmid a b$.

(i) When $a$ and $b$ are not both odd.

Since $a$ and $b$ are of opposite parity we can pick our $x_{0}, y_{0}$ to both be odd (indeed either $\left(x_{0}, y_{0}\right)$ or $\left(x_{0}+b, y_{0}+a\right)$ will be of this form). Hence on the line segment $n\left(l_{3} \cap T\right)$ our parametrisation gives $(-1)^{x+y}=(-1)^{t}$, and, writing

$$
f_{n}(t):=Q\left(n x_{0}+b t, n y_{0}+a t\right)^{-s},
$$

we have

$$
A_{N}(T, Q, s)=\sum_{1 \leq n \leq N} A_{n}\left(l_{3} \cap T, Q, s\right),
$$

where

$$
A_{n}\left(l_{3} \cap T, Q, s\right)=\sum_{n \alpha_{1} \leq t \leq n \alpha_{2}}(-1)^{t} f_{n}(t) .
$$

We first observe some elementary bounds on $f_{n}$ :

$$
\begin{aligned}
\left|f_{n}(t)\right| & =\left|Q(x, y)^{-s}\right|=O\left(n^{-2 \sigma}\right), \\
\left|f_{n}^{\prime}(t)\right| & =\left|-s((2 \alpha b+\beta a) x+(2 \gamma a+\beta b) y) Q(x, y)^{-s-1}\right|=O\left(n^{-2 \sigma-1}\right), \\
\left|f_{n}^{\prime \prime}(t)\right| & =\left|((2 \alpha b+\beta a) x+(2 \gamma a+\beta b) y)^{2} \frac{(s+1)}{Q(x, y)^{s+2}}-2 s \frac{Q(a, b)}{Q(x, y)^{s+2}}\right| \\
& =O\left(n^{-2 \sigma-2}\right) .
\end{aligned}
$$

Pairing odd and even $t$, we have

$$
A_{n}\left(l_{3} \cap T, Q, s\right)=M_{1}+M_{2},
$$

where

and

$$
M_{1}:=\sum_{\frac{1}{2} n \alpha_{1} \leq t \leq \frac{1}{2} n \alpha_{2}}\left(f_{n}(2 t)-f_{n}(2 t+1)\right)
$$

$$
M_{2}:=u_{2}(n) f_{n}\left(2 t_{n, 2}+1\right)-u_{1}(n) f_{n}\left(2 t_{n, 1}+1\right),
$$


where $u_{1}(n):=1$ if there is an integer $t_{n, 1}$ in $\left[\frac{n A_{1}}{2 B_{1}}-\frac{1}{2}, \frac{n A_{1}}{2 B_{1}}\right)$ and 0 otherwise, and $u_{2}(n):=1$ if there is an integer $t_{n, 2}$ in $\left(\frac{n A_{2}}{2 B_{2}}-\frac{1}{2}, \frac{n A_{2}}{2 B_{2}}\right]$ and 0 otherwise.

Using the bound for $f_{n}^{\prime \prime}(t)$, we have

$$
f_{n}(2 t+1)-f_{n}(2 t)=\int_{2 t}^{2 t+1} f_{n}^{\prime}(u) d u=\int_{2 t}^{2 t+1}\left(f_{n}^{\prime}(2 t)+O\left(n^{-2 \sigma-2}\right)\right) d u .
$$

This and the observation that for a differentiable function $g(x)$

$$
\begin{aligned}
\sum_{x_{1}<n \leq x_{2}} g(n) & =\left[x_{2}\right] g\left(\left[x_{2}\right]\right)-\left[x_{1}\right] g\left(\left[x_{1}\right]\right)-\int_{\left[x_{1}\right]}^{\left[x_{2}\right]}[u] g^{\prime}(u) d u \\
& =\int_{\left[x_{1}\right]}^{\left[x_{2}\right]}\left(g(u)-\{u\} g^{\prime}(u)\right) d u
\end{aligned}
$$

enable us to evaluate $M_{1}$ :

$$
\begin{aligned}
M_{1} & =-\frac{1}{2} \sum_{\frac{1}{2} n \alpha_{1} \leq t \leq \frac{1}{2} n \alpha_{2}}\left(\frac{d}{d t} f_{n}(2 t)+O\left(n^{-2 \sigma-2}\right)\right) \\
& =-\frac{1}{2} \int_{\left[\frac{1}{2} n \alpha_{1}\right]}^{\left[\frac{1}{2} n \alpha_{2}\right]}\left(\frac{d}{d t} f_{n}(2 t)+O\left(n^{-2 \sigma-2}\right)\right) d t+O\left(n^{-2 \sigma-1}\right) \\
& =-\frac{1}{2}\left(f_{n}\left(2 \frac{1}{2} n \alpha_{2}\right)-f_{n}\left(2 \frac{1}{2} n \alpha_{1}\right)\right)+O\left(n^{-2 \sigma-1}\right) \\
& =\frac{1}{2} Q\left(n P_{1}\right)^{-s}-\frac{1}{2} Q\left(n P_{2}\right)^{-s}+O\left(n^{-2 \sigma-1}\right) .
\end{aligned}
$$

For $M_{2}$ (approximating the $\left(2 t_{n, i}+1\right)$ by $\left.n \alpha_{i}\right)$ we have

$$
u_{i}(n) f\left(2 t_{i, n}+1\right)=u_{i}(n) Q\left(n P_{i}\right)^{-s}+O\left(n^{-2 \sigma-1}\right),
$$

giving

$A_{n}\left(l_{3} \cap T, Q, s\right)=\frac{1}{2} Q\left(P_{1}\right)^{-s} \frac{\left(1-2 u_{1}(n)\right)}{n^{2 s}}-\frac{1}{2} Q\left(P_{2}\right)^{-s} \frac{\left(1-2 u_{2}(n)\right)}{n^{2 s}}+O\left(n^{-2 \sigma-1}\right)$.

Notice that

$$
A_{n}\left(l_{3} \cap T, Q, s\right)=O\left(n^{-2 \sigma}\right) .
$$

Hence

$$
\begin{aligned}
A_{N}(T, Q, s)= & \frac{1}{2} Q\left(P_{1}\right)^{-s} \sum_{n \leq N} \frac{\left(1-2 u_{1}(n)\right)}{n^{2 s}}-\frac{1}{2} Q\left(P_{2}\right)^{-s} \sum_{n \leq N} \frac{\left(1-2 u_{2}(n)\right)}{n^{2 s}} \\
& +C_{1}(s)+O\left(\frac{1}{N^{2 \sigma}}\right),
\end{aligned}
$$

where $C_{1}(s)$ is analytic for all $\operatorname{Re} s>0$. Noting that the functions $u_{i}(n)$ are defined modulo $\left(2 B_{i}\right)$ and that for a function $u(n) \leq 1$ defined modulo $q$

$$
\begin{aligned}
\sum_{k q \leq n<(k+1) q} u(n) n^{-2 s} & =(k q)^{-2 s} \sum_{k q \leq n<(k+1) q} u(n)+O\left(q k^{-2 \sigma-1}\right) \\
& =\left(\frac{1}{q} \sum_{m=1}^{q} u(m)\right)\left(\sum_{k q \leq n<(k+1) q} n^{-2 s}\right)+O\left(q k^{-2 \sigma-1}\right),
\end{aligned}
$$


we can plainly write

$$
\sum_{1 \leq n \leq N} \frac{\left(1-2 u_{i}(n)\right)}{n^{2 s}}=\left(1-\frac{1}{B_{i}} \sum_{m=1}^{2 B_{i}} u_{i}(m)\right)\left(\sum_{1 \leq n \leq N} n^{-2 s}\right)+C_{2, i}(s)+O\left(N^{-2 \sigma}\right)
$$

with $C_{2, i}(s)$ analytic in $\operatorname{Re} s>0$. Now

$$
\begin{aligned}
\sum_{m=1}^{2 B_{1}} u_{1}(n) & =\#\left\{1 \leq n \leq 2 B_{1}: n A_{1} \equiv 1,2, \ldots, \text { or } B_{1}\left(\bmod 2 B_{1}\right)\right\} \\
& = \begin{cases}B_{1} & \text { if } 2 \nmid A_{1}, \\
B_{1}-1 & \text { if } 2 \mid A_{1},\end{cases}
\end{aligned}
$$

and

$$
\begin{aligned}
\sum_{m=1}^{2 B_{2}} u_{2}(n) & =\#\left\{1 \leq n \leq 2 B_{2}: n A_{2} \equiv 0,1, \ldots, \text { or }\left(B_{2}-1\right)\left(\bmod 2 B_{2}\right)\right\} \\
& = \begin{cases}B_{2} & \text { if } 2 \nmid A_{2}, \\
B_{2}-1 & \text { if } 2 \mid A_{2} .\end{cases}
\end{aligned}
$$

Noting that (since $x_{0}$ and $y_{0}$ are both odd) $2 \mid A_{i}$ exactly when $2 \nmid r_{i} s_{i}$ we obtain

$$
\begin{aligned}
& A_{N}(T, Q, s) \\
& =\frac{1}{2} \frac{\lambda\left(l_{1}\right)}{B_{1}} Q\left(P_{1}\right)^{-s} \sum_{n \leq N} n^{-2 s}+\frac{1}{2} \frac{\lambda\left(l_{2}\right)}{B_{2}} Q\left(P_{2}\right)^{-s} \sum_{n \leq N} n^{-2 s}+C_{3}(s)+O\left(N^{-2 \sigma}\right),
\end{aligned}
$$

where

$$
\lambda\left(l_{i}\right)= \begin{cases}1 & \text { if } 2 \nmid r_{i} s_{i}, \\ 0 & \text { if } 2 \mid r_{i} s_{i}\end{cases}
$$

and $C_{3}(s)$ is analytic for $\operatorname{Re} s>0$. Since we have already shown that

$$
A_{N}\left(l_{3} \cap T, Q, s\right)=O\left(N^{-2 \sigma}\right),
$$

it will be enough to show that for $i=1,2$

$$
A_{N}\left(l_{i} \cap T, Q, s\right)=\frac{\lambda\left(l_{i}\right)}{B_{i}} Q\left(P_{i}\right)^{-s} \sum_{n \leq N} n^{-2 s}+C_{4, i}(s)+O\left(N^{-2 \sigma}\right),
$$

for some suitable analytic $C_{4, i}(s)$.

Since $N\left(l_{i} \cap T\right) \cap \mathbf{Z}^{2}=\left\{n B_{i} P_{i}: 1 \leq n \leq N / B_{i}\right\}$, we have

$$
A_{N}\left(l_{i} \cap T, Q, s\right)=Q\left(B_{i} P_{i}\right)^{-s} \sum_{n \leq N / B_{i}}(-1)^{r_{i}+s_{i}} n^{-2 s} .
$$

Now if $\lambda\left(l_{i}\right)=0$ we have $r_{i}+s_{i}$ odd and

$$
\begin{aligned}
A_{N}\left(l_{i} \cap T, Q, s\right) & =Q\left(B_{i} P_{i}\right)^{-s} \sum_{n \leq N / B_{i}}(-1)^{n} n^{-2 s} \\
& =Q\left(B_{i} P_{i}\right)^{-s} \sum_{t \leq N / 2 B_{i}}\left((2 t)^{-2 s}-(2 t-1)^{-2 s}\right)+O\left(N^{-2 \sigma}\right) \\
& =\sum_{t \leq N / B_{i}} O\left(t^{-2 \sigma-1}\right)+O\left(N^{-2 \sigma}\right) \\
& =C_{4, i}(s)+O\left(N^{-2 \sigma}\right)
\end{aligned}
$$


while if $\lambda\left(l_{i}\right)=1$ then $r_{i}+s_{i}$ is even and

$$
\begin{aligned}
& \frac{Q\left(P_{i}\right)^{-s}}{B_{i}} \sum_{n \leq N} n^{-2 s} \\
& =\frac{Q\left(P_{i}\right)^{-s}}{B_{i}} \sum_{n \leq N / B_{i}} \sum_{l=1}^{B_{i}}\left(n B_{i}+l\right)^{-2 s}+O\left(N^{-2 \sigma}\right) \\
& =\frac{Q\left(P_{i}\right)^{-s}}{B_{i}} \sum_{n \leq N / B_{i}} \sum_{l=1}^{B_{i}}\left(n B_{i}\right)^{-2 s}+\sum_{n \leq N / B_{i}} O\left(n^{-2 \sigma-1}\right)+O\left(N^{-2 \sigma}\right) \\
& =Q\left(B_{i} P_{i}\right)^{-s} \sum_{n \leq N / B_{i}} n^{-2 s}+C_{5, i}(s)+O\left(N^{-2 \sigma}\right),
\end{aligned}
$$

where the $C_{4, i}(s)$ and $C_{5, i}(s)$ are analytic for $\operatorname{Re} s>0$, as was required.

\section{(ii) When both $a$ and $b$ are odd.}

When $a$ and $b$ are both odd any $x_{0}, y_{0}$ satisfying $a x_{0}-b y_{0}=1$ are necessarily of opposite parity, so that on the line $a x-b y=n$ our parametrisation gives $(-1)^{x+y}=$ $(-1)^{n}$. We here choose our $x_{0}, y_{0}$ to satisfy $A_{i}=r_{i} x_{0}-s_{i} y_{0}>0$ for $i=1,2$ (this we can do by replacing $x_{0}, y_{0}$ by $x_{0}+b j, y_{0}+a j$ for a suitably small $j$ ), and set

$$
\beta_{i}=\frac{B_{i}}{A_{i}}
$$

Hence, altering the order of the $n$ and $t$ summations,

$$
\begin{aligned}
A_{N}(T, Q, s) & =\sum_{n \leq N}(-1)^{n} \sum_{n \alpha_{1} \leq t \leq n \alpha_{2}} f_{n}(t) \\
& =\sum_{\alpha_{1} \leq t \leq N \alpha_{1}} E_{1}+\sum_{N \alpha_{1} \leq t \leq N \alpha_{2}} E_{2},
\end{aligned}
$$

where

$$
\begin{aligned}
& E_{1}:=\sum_{t \beta_{2} \leq n \leq t \beta_{1}}(-1)^{n} f_{n}(t), \\
& E_{2}:=\sum_{t \beta_{2} \leq n \leq N}(-1)^{n} f_{n}(t) .
\end{aligned}
$$

Just as in case (i) (with the roles of $n$ and $t$ reversed), summing along the line joining $t \beta_{1} P_{1}$ and $t \beta_{2} P_{2}$, we have

$$
\begin{aligned}
& E_{1}=\frac{1}{2} Q\left(\beta_{2} P_{2}\right)^{-s} \frac{\left(1-2 v_{2}(t)\right)}{t^{2 s}}-\frac{1}{2} Q\left(\beta_{1} P_{1}\right)^{-s} \frac{\left(1-2 v_{1}(t)\right)}{t^{2 s}}+O\left(t^{-2 \sigma-1}\right) \\
& E_{2}=\frac{1}{2} Q\left(\frac{B_{2}}{A_{2}} P_{2}\right)^{-s}\left(1-2 v_{2}(t)\right) t^{-2 s}-\frac{1}{2} f_{N}(t)(1-2 w(N)) t^{-2 s}+O\left(t^{-2 \sigma-1}\right)
\end{aligned}
$$

where $v_{1}(n)=1$ if there is an integer in $\left(\frac{t B_{1}}{2 A_{1}}-\frac{1}{2}, \frac{t B_{1}}{2 A_{1}}\right]$ and 0 otherwise, $v_{2}(n)=1$ if there is an integer in $\left[\frac{t B_{2}}{2 A_{2}}-\frac{1}{2}, \frac{t B_{2}}{2 A_{2}}\right)$ and 0 otherwise, and $w(N)$ is 1 if $N$ is even 
and 0 if $N$ is odd. Hence

$$
\begin{aligned}
A_{N}(T, Q, s)= & \frac{1}{2} \sum_{N \alpha_{1} \leq t \leq N \alpha_{2}}(-1)^{N} f_{N}(t)+\frac{1}{2} Q\left(\beta_{2} P_{2}\right)^{-s} \sum_{\alpha_{1} \leq t \leq N \alpha_{1}} \frac{\left(1-2 v_{2}(t)\right)}{t^{2 s}} \\
& +\frac{1}{2} Q\left(\beta_{1} P_{1}\right)^{-s} \sum_{\alpha_{1} \leq t \leq N \alpha_{1}} \frac{\left(2 v_{1}(t)-1\right)}{t^{2 s}}+C_{6}(s)+O\left(N^{-2 \sigma}\right),
\end{aligned}
$$

with $C_{6}(s)$ analytic in $\operatorname{Re} s>0$. It is not hard to see that the first sum is simply $\frac{1}{2} A_{N}\left(l_{3} \cap T, Q, s\right)$. Hence it remains to verify that the other sums differ from $\frac{1}{2} A_{N}\left(l_{2} \cap T, Q, s\right)$ and $\frac{1}{2} A_{N}\left(l_{1} \cap T, Q, s\right)$ by a function analytic for Re $s>0$. One proceeds just as in case (i) (with the roles of $A_{i}$ and $B_{i}$ reversed and with $2 \mid B_{i}$ if and only if $2 \nmid r_{i} s_{i}$ ), showing that for $i=1,2$

$$
\begin{aligned}
(-1)^{i} Q\left(\beta_{i} P_{i}\right)^{-s} & \sum_{\alpha_{i} \leq t \leq N \alpha_{i}} \frac{\left(1-2 v_{i}(t)\right)}{t^{2 s}} \\
& =\frac{\lambda\left(l_{i}\right)}{A_{i}} Q\left(\beta_{i} P_{i}\right)^{-s} \sum_{\alpha_{i} \leq t \leq N \alpha_{i}} t^{-2 s}+C_{7, i}(s)+O\left(N^{-2 \sigma}\right) \\
& =\lambda\left(l_{i}\right) Q\left(B_{i} P_{i}\right)^{-s} \sum_{1 / B_{i} \leq t \leq N / B_{i}} t^{-2 s}+C_{8, i}(s)+O\left(N^{-2 \sigma}\right) \\
& =A_{N}\left(l_{i} \cap T, Q, s\right)+C_{9, i}(s)+O\left(N^{-2 \sigma}\right),
\end{aligned}
$$

with $C_{9, i}(s)$ analytic in $\operatorname{Re} s>0$, and the result is plain.

Proof of Theorem 7. Part (i): Define $d$ to be the least common multiple of the $d_{i}$ and (for a given positive real $\nu$ ) set $N=d[\nu / d]$. Then $\nu P$ and $N P$ differ by at most a finite collection of lines of the form $l_{i}: a_{i} x-b_{i} y=c_{i} n, c_{i} \neq 0$, with $N \leq n \leq \nu$. Hence $A_{\nu}(P, Q, s)$ differs from $A_{N}^{*}(P, Q, s)$ by a finite sum of $\frac{1}{2} A_{n}\left(l_{i} \cap P, Q, s\right)$ with $c_{i} \neq 0$ and $N \leq n \leq \nu$, exactly $\frac{1}{2} A_{N}\left(l_{i} \cap P, Q, s\right)$ for any radial lines (i.e. lines with $\left.c_{i}=0\right)$, together with a finite set of points lying at the intersections of these various lines.

However, we have already seen (recall (9)) that when $a_{i} / d_{i}$ and $b_{i} / d_{i}$ are of opposite parity the lines $a_{i} x+b_{i} y=n c_{i}, c_{i} \neq 0$, contribute $O\left(\left(c_{i} n / d_{i}\right)^{-2 \sigma}\right)=$ $O\left(\nu^{-2 \sigma}\right)$, while for the radial lines we showed (see (10)) a contribution $C_{i}(s)+$ $O\left(N^{-2 \sigma}\right)$ (with $C_{i}(s)$ analytic for Re $s>0$ and $C_{i}(s)=0$ unless $(0,0)$ lies on $\left.l_{i} \cap P\right)$. The left-over points, being of distance $\asymp \nu$ from the origin, similarly contribute only terms of size $O\left(\nu^{-2 \sigma}\right)$, and the limit exists for all Re $s>0$.

Part (ii): Given our polygon with sides $l_{i}: a_{i} x-b_{i} y=c_{i}$, we set

$$
\delta=\frac{1}{2} \min _{i}\left|c_{i}\right|^{-1}
$$

and observe that for an integer $N$ going from $N P$ to $(N+\delta) P$ we may lose some lines of points but can gain no new lattice points, and similarly going from $(N-\delta) P$ to $N P$ we may gain but cannot lose lattice points. Hence the two differences

$$
\left|A_{N \pm \delta}(P, Q, \delta)-A_{N}(P, Q, \delta)\right|
$$

consist solely of sums of $A_{N}\left(l_{i} \cap P, Q, \sigma\right)$ with $c_{i} \neq 0$ (together with odd points of intersection that are of size $O\left(N^{-2 \sigma}\right)$ ). Further if we take $N=2 n d$ (where $d$ is the least common multiple of all the $\left.\operatorname{gcd}\left(a_{i}, b_{i}\right)\right)$ then every $l_{i}$ with $c_{i} \neq 0$ will appear 
in one of these sums (which one will depend on whether $(0,0)$ lies on the same or opposite side of the line as the interior of the polygon). As in part (i), the lines $l_{i}$ with $a_{i} / d_{i}$ and $b_{i} / d_{i}$ of opposite parity and $c_{i} \neq 0$ will only contribute $O\left(N^{-2 \sigma}\right)$. However, if $a_{I} / d_{I}$ and $b_{I} / d_{I}$ are both odd and $c_{I} \neq 0$, then

$$
A_{N}\left(l_{I} \cap T, Q, \sigma\right)=(-1)^{\left(c_{i}(2 n d) / d_{I}\right)} \sum_{\kappa_{1} N \leq t \leq \kappa_{2} N} f_{n}(t)
$$

will contribute

$$
\sum_{\kappa_{1} N \leq t \leq \kappa_{2} N} f_{n}(t) \geq e_{1} \sum_{\kappa_{1} N \leq t \leq \kappa_{2} N} N^{-2 \sigma} \geq e_{2} N^{1-2 \sigma},
$$

for some positive constants $e_{i}$. Hence if $0<\sigma \leq 1 / 2$ and we have at least one $l_{I}$ with $a_{I} / d_{I}$ and $b_{I} / d_{I}$ both odd and $c_{I} \neq 0$, then at least one of the two differences $\left|A_{N \pm \delta}(P, Q, \delta)-A_{N}(P, Q, \delta)\right|$ will always be bounded away from 0 by a constant (irrespective of $N)$ and the limit $A(P, Q, \sigma)$ cannot exist. When $P$ is a star body centred at $(0,0)$ we set $\delta=1$, and (since we only gain points in going from $(N-1) P$ to $P)$ the same argument shows that $A_{N}(P, Q, \sigma)-A_{N-1}(P, Q, \sigma)$ does not tend to zero with $N$; hence the limit does not exist even if we restrict ourselves (as is natural) just to integer scalings of $P$. When all the $l_{i}$ with $c_{i} \neq 0$ have $a_{i} / d_{i}, b_{i} / d_{i}$ of opposite parity but there are radial lines ( $l_{1}$ through $l_{k}$ say) with $c_{i}=0$ and $a_{i} / d_{i}, b_{i} / d_{i}$ both odd we consider $A_{N}(P, Q, \sigma)$ for $N=n d$. By the theorem it is clear that convergence for $0<\sigma \leq 1 / 2$ will be determined solely by the sum over the perimeter. As in part (i) the lines $l_{i}, i>k$, cannot disturb the convergence. However the radial lines $l_{i}, i=1, \ldots, k$, each contribute

$$
\begin{aligned}
A_{N}\left(l_{i} \cap P, Q, \sigma\right) & =\sum_{\kappa_{1} N \leq t \leq \kappa_{2} N} f_{n}(t)=f\left(P_{i}\right) \sum_{\substack{t \neq 0 \\
\kappa_{1} N \leq t \leq \kappa_{2} N}} t^{-2 \sigma} \\
& \geq e_{3} \begin{cases}\log N & \text { if } \sigma=1 / 2, \\
N^{1-2 \sigma} & \text { if } \sigma<1 / 2\end{cases}
\end{aligned}
$$

(where $e_{3}$ is some positive constant), and the resulting sum is plainly unbounded as $N \rightarrow \infty$.

Corollary 3 is immediate from Theorem 7.

Proof of Theorem 8. We split $P$ into a series of cones $P_{i}$ with base a face of $P$ and vertex $(0,0,0)$ :

$$
P_{i}:=\left\{(x, y, z): a_{i} x+b_{i} y+c_{i} z \leq e_{i}, \alpha_{i j} x+\beta_{i j} y+\gamma_{i j} z \leq 0,1 \leq j \leq J_{i}\right\},
$$

for some integers $\alpha_{i j}, \beta_{i j}, \gamma_{i j}$, so that

$$
A_{N}(P, Q, s)=\sum_{i=1}^{I} A_{N}^{\star}\left(P_{i}, Q, s\right)
$$

where $A_{N}^{\star}(P, Q, s)$ indicates that points on the sides of $N P$ (excepting the base) are to be counted with weight $1 / 2$. Slicing up each three dimensional polygon $N P_{i}$ into two dimensional polygons $P_{i, m}$ parallel to its base,

$$
P_{i, m}:=\left\{(x, y, z) \in \mathbf{Z}^{2}: a_{i} x+b_{i} y+c_{i} z=m, \alpha_{i j} x+\beta_{i j} y+\gamma_{i j} z \leq 0,1 \leq j \leq J_{i}\right\}
$$


we have

$$
A_{N}^{\star}\left(P_{i}, Q, s\right)=\sum_{\substack{m=1 \\ d_{i} \mid m}}^{N e_{i}} \sum_{\vec{x} \in P_{i, m}}^{*} Q(\vec{x})^{-s}
$$

where $\sum^{*}$ denotes that points on the boundary of the polygon are to be counted with weight $1 / 2$.

We suppose now that $P_{i, m}$ comes from a face with at least one of $a_{i} / d_{i}, b_{i} / d_{i}$ or $c_{i} / d_{i}$ even. We assume (replacing $m$ by $d_{i} m$ as necessary) that $\operatorname{gcd}\left(a_{i}, b_{i}, c_{i}\right)=1$, and (reordering as necessary) that $c_{i}$ is odd and $a_{i}$ is even. Setting $\alpha_{i}=\operatorname{gcd}\left(a_{i}, c_{i}\right)$ and choosing an even integer $\overline{b_{i}}$ such that $b_{i} \overline{b_{i}} \equiv 1\left(\bmod \alpha_{i}\right)$ and an odd integer $\overline{\left(a_{i} / \alpha_{i}\right)}$ such that $\left(a_{i} / \alpha_{i}\right) \overline{\left(a_{i} / \alpha_{i}\right)} \equiv 1\left(\bmod c_{i} / \alpha_{i}\right)$, we make the change of variables demanded by the relation $a_{i} x+b_{i} y \equiv m\left(\bmod c_{i}\right)$ on $P_{i, m}$ :

$$
y=\overline{b_{i}} m+\alpha_{i} y^{\prime}
$$

and

$$
x=\overline{\left(\frac{a_{i}}{\alpha_{i}}\right)}\left(\frac{1-b_{i} \overline{b_{i}}}{\alpha_{i}}\right) m-\overline{\left(\frac{a_{i}}{\alpha_{i}}\right)} b_{i} y^{\prime}+\left(\frac{c_{i}}{\alpha_{i}}\right) x^{\prime} .
$$

Observe that $a_{i} x+b_{i} y+c_{i} z=m$ becomes

$$
z=m\left(\frac{1-b_{i} \overline{b_{i}}}{\alpha_{i}}\right)\left(\frac{1-\left(\frac{a_{i}}{\alpha_{i}} \overline{\left(\frac{a_{i}}{\alpha_{i}}\right)}\right.}{c_{i} / \alpha_{i}}\right)-\left(\frac{a_{i}}{\alpha_{i}}\right) x^{\prime}-b_{i}\left(\frac{1-\left(\frac{a_{i}}{\alpha_{i}}\right) \overline{\left(\frac{a_{i}}{\alpha_{i}}\right)}}{c_{i} / \alpha_{i}}\right) y^{\prime},
$$

and that

$$
(-1)^{x+y+z}=(-1)^{x^{\prime}+y^{\prime}} .
$$

Hence the sum of $(x, y, z)$ over $P_{i, m}$ is replaced by a sum of $\left(x^{\prime}, y^{\prime}\right)$ over $m R_{i} \cap \mathbf{Z}^{2}$, where $R_{i}$ is the polygon

$$
R_{i}=\left\{\left(x^{\prime}, y^{\prime}\right) \in \mathbf{Z}^{2}: \alpha_{i j}^{\prime} x^{\prime}+\beta_{i j}^{\prime} y^{\prime} \leq \gamma_{i j}^{\prime}, 1 \leq j \leq J_{i}\right\},
$$

and the $\alpha_{i j}^{\prime}, \beta_{i j}^{\prime}, \gamma_{i j}^{\prime}$ are integers with, we shall assume, no common factor and $d_{i j}:=\operatorname{gcd}\left(\alpha_{i j}^{\prime}, \beta_{i j}^{\prime}\right)$. Writing

$$
Q(x, y, z)=Q_{i}\left(x^{\prime}, y^{\prime}, m\right)
$$

(where $Q_{i}(x, y, z)$ will be a positive definite quadratic form), we have

$$
\sum_{(x, y, z) \in P_{i, m}}^{*} Q(x, y, z)^{-s}=\sum_{(x, y) \in m R_{i} \cap \mathbf{Z}^{2}}^{*}(-1)^{x+y} Q_{i}(x, y, m)^{-s}=: A_{m}^{*}\left(R_{i}, Q_{i}, s\right),
$$

with $\sum^{*}$ denoting that points on the perimeter are counted with weight $1 / 2$. To evaluate the $A_{m}^{*}\left(R_{i}, Q_{i}, s\right)$ one proceeds almost exactly as in the proof of Proposition 1 (replacing the bounds for the $k$ th derivative, $k=0,1,2$, in (8) by

$$
\left|f_{n}^{(k)}(t)\right|=O\left((|n|+|m|)^{-2 \sigma-k}\right)
$$

and so on) to obtain

$$
\sum_{(x, y) \in m R_{i} \cap \mathbf{Z}^{2}}^{\dagger}(-1)^{x+y} Q_{i}(x, y, m)^{-s}=O\left(m^{-2 \sigma}\right),
$$


where $\sum^{\dagger}$ denotes that for each side $l_{i j}$ of the polygon $R_{i}$ the last line of points parallel to that side,

$$
\mathcal{L}_{i j}(m):=\left[m \frac{\gamma_{i j}^{\prime}}{d_{i j}}\right] R_{i j}, \quad R_{i j}:=\frac{d_{i j}}{\gamma_{i j}^{\prime}}\left(l_{i j} \cap R_{i}\right),
$$

is to be included in the sum with weight $1 / 2$. The desired sum $A^{*}\left(R_{i}, Q_{i}, s\right)$ thus differs from this latter sum only by the addition or exclusion of the (half-weighted) last lines of those sides $l_{i j}$ for which $m \neq \equiv 0\left(\bmod d_{i j}\right)$, and

$$
A_{m}^{*}\left(R_{i}, Q_{i}, s\right)=O\left(m^{-2 \sigma}\right)+\frac{1}{2} \sum_{\substack{j=1 \\ d_{i j \nmid m}}}^{J_{i}} A_{i j}(m),
$$

where

$$
A_{i j}(m):= \pm \sum_{(x, y) \in \mathcal{L}_{i j}(m)}(-1)^{x+y} Q_{1}(x, y, m)^{-s}
$$

with the \pm sign determined by whether $(0,0)$ lies on the interior or exterior side of $l_{i j}$ respectively. Thus

$$
A_{N}^{\star}\left(P_{i}, Q, s\right)=C_{i, 0}(s)+\sum_{j=1}^{J_{i}} E_{i j}+O\left(N^{1-2 \sigma}\right),
$$

with

$$
E_{i j}:=\sum_{\substack{1 \leq m \leq N e_{i} / d_{i} \\ d_{i j} \nmid m}} A_{i j}(m)
$$

and $C_{i, 0}(s)$ analytic for all $\operatorname{Re} s>1 / 2$.

Now if $\alpha_{i j}^{\prime \prime}:=\alpha_{i j}^{\prime} / d_{i j}$ and $\beta_{i j}^{\prime \prime}:=\beta_{i j}^{\prime} / d_{i j}$ are of opposite parity, one readily shows that alternation in sign along the line $\mathcal{L}_{i j}(m)$ gives (in the manner of (9))

$$
A_{i j}(m)=O\left(m^{-2 \sigma}\right)
$$

and hence

$$
E_{i j}=C_{i j}(s)+O\left(N^{1-2 \sigma}\right)
$$

with the $C_{i j}(s)$ analytic for $\operatorname{Re} s>1 / 2$.

Now if the $\alpha_{i j}^{\prime \prime}, \beta_{i j}^{\prime \prime}$ are both odd, parametrising the line (as in the proof of Proposition 1), we have

$$
\begin{aligned}
A_{n}\left(R_{i j}, Q_{i}(m), s\right) & :=\sum_{(x, y) \in n R_{i j} \cap \mathbf{Z}^{2}}(-1)^{x+y} Q_{i}(x, y, m)^{-s} \\
& =(-1)^{n} \sum_{n \xi_{1} \leq t \leq n \xi_{2}} Q_{1}\left(n x_{0}-\alpha_{i j}^{\prime \prime}, \beta_{i j}^{\prime \prime} t-n y_{0}, m\right)^{-s},
\end{aligned}
$$

for some fixed integers $x_{0}=x_{0}(i, j), y_{0}=y_{0}(i, j)\left(\right.$ with $\left.\alpha_{i j}^{\prime \prime} x_{0}-\beta_{i j}^{\prime \prime} y_{0}=1\right)$ and rational numbers $\xi_{k}=\xi_{k}(i, j)$. So for bounded integers $k_{1}$ and $k_{2}$ we certainly have

$$
A_{n+k_{1}}\left(R_{i j}, Q_{i}\left(m+k_{2}\right), s\right)=(-1)^{k_{1}} A_{n}\left(R_{i j}, Q_{i}(m), s\right)+O\left((|m|+|n|)^{-2 \sigma}\right),
$$

where a trivial bound gives

$$
A_{n}\left(R_{i j}, Q_{1}(m), s\right)=O\left(\frac{n}{(|n|+|m|)^{2 \sigma}}\right) .
$$


Hence, splitting the sum over $m$ into multiples of $2 d_{i j}$, we have

$$
E_{i j}=S_{i j} \sum_{l \leq N e_{i} / 2 d_{i} d_{i j}} A_{i j}\left(2 d_{i j} l\right)+\sum_{1 \leq m \leq N e_{i} / d_{i}} O\left(m^{-2 \sigma}\right)+O\left(N^{1-2 \sigma}\right),
$$

where (as may be readily checked)

$$
S_{i j}:=\sum_{\substack{m=1 \\ d_{i j} \nmid m}}^{2 d_{i j}}(-1)^{\left[m \gamma_{i j}^{\prime} / d_{i j}\right]}=0
$$

giving

$$
E_{i j}=C_{i j}(s)+O\left(N^{1-2 \sigma}\right),
$$

with $C_{i j}(s)$ analytic for $\operatorname{Re} s>1 / 2$.

Therefore

$$
A_{N}^{\star}\left(P_{i}, Q, s\right)=C_{i}(s)+O\left(N^{1-2 \sigma}\right)
$$

(where $C_{i}(s)$ is analytic for all $\operatorname{Re} s>1 / 2$ ), and the result (i) follows at once if all the faces have at least one $a_{i} / d_{i}, b_{i} / d_{i}, c_{i} / d_{i}$ even.

For part (ii) one proceeds in the manner of the proof of Theorem 2 to show, by counting the number of points on the faces with $a_{i} / d_{i}, b_{i} / d_{i}, c_{i} / d_{i}$ all odd, that (for suitable multiples $N$ and $\delta:=\frac{1}{2} \min _{i} e_{i}^{-1}$ ) the contribution from those faces to $\left|A_{N}(P, Q, s)-A_{N-\delta}(P, Q, s)\right|$ does not tend to zero as $N \rightarrow \infty$.

Proof of Theorem 9. The approach resembles the proof of Theorems 5 and 6 in Borwein-Borwein-Taylor $[1, \S I V]$. We set

$$
\begin{aligned}
T(z) & :=\sum_{n_{1}=-\infty}^{\infty} \cdots \sum_{n_{k}=-\infty}^{\infty} q\left(n_{1}, \ldots, n_{k}\right) z^{Q\left(n_{1}, \ldots, n_{k}\right)}, \\
T_{\vec{m}}(z) & :=\sum_{n_{1}=-m_{1}}^{m_{1}} \ldots \sum_{n_{k}=-m_{k}}^{m_{k}} q\left(n_{1}, \ldots, n_{k}\right) z^{Q\left(n_{1}, \ldots, n_{k}\right)},
\end{aligned}
$$

and define the normalized Mellin transform $M_{s}(f)$ for Re $s>0$ by

$$
M_{s}(f):=\Gamma^{-1}(s) \int_{0}^{\infty} f(t) t^{s-1} d t
$$

We set

$$
F(s):=M_{s}\left(T\left(e^{-t}\right)-q(0, \ldots, 0)\right)
$$

and observe that (since $M_{s}\left(e^{-a t}\right)=a^{-s}$ for $a>0$ )

$$
A_{\vec{m}}(s)=M_{s}\left(T_{\vec{m}}\left(e^{-t}\right)-q(0, \ldots, 0)\right) .
$$

We shall need the following uniform boundedness lemma:

Lemma 3. For any $t>0$ and integers $N_{i} \geq 0$ (with at least one $N_{i}>0$ ),

$$
S:=\left|\sum_{n_{1}=N_{1}}^{\infty} \cdots \sum_{n_{k}=N_{k}}^{\infty} q\left(n_{1}, \ldots, n_{k}\right) e^{-t Q\left(n_{1}, \ldots, n_{k}\right)}\right|<C e^{-\lambda\left(N_{1}^{2}+\cdots+N_{k}^{2}\right) t}
$$

for some $C=C(Q)>0$ and $\lambda=\lambda(Q)>0$. 
Proof. We set

$$
g\left(u_{1}, \ldots, u_{k}\right):=e^{-Q\left(u_{1}, \ldots, u_{k}\right)}
$$

and write $\chi_{\vec{N}}$ for the characteristic function of the region $\left\{\left(u_{1}, \ldots, u_{k}\right) \in \mathbf{R}^{k}\right.$ : $\left.u_{i} \geq N_{i}\right\}$. Applying the partial summation technique employed in the proof of Theorem 1 ,

$$
\begin{aligned}
\sum_{n=-\infty}^{\infty} a(n) b(n) & =\sum_{n=-\infty}^{\infty}\left(\sum_{l=-\infty}^{n} a(l)\right)(b(n)-b(n+1)) \\
& =-\sum_{n=-\infty}^{\infty}\left(\sum_{l=-\infty}^{n} a(l)\right) \int_{n}^{n+1} b^{\prime}(u) d u
\end{aligned}
$$

to each of the variables $n_{i}$ in turn, we obtain

$$
S=\left|\sum_{n_{1}=-\infty}^{\infty} \cdots \sum_{n_{k}=-\infty}^{\infty} W\left(n_{1}, \ldots, n_{k}\right) I\left(n_{1}, \ldots, n_{k}\right)\right|
$$

where

$$
W\left(n_{1}, \ldots, n_{k}\right):=\sum_{r_{1}=-\infty}^{n_{1}} \cdots \sum_{r_{k}=-\infty}^{n_{k}} q\left(r_{1}, \ldots, r_{k}\right) \chi_{\vec{N}}\left(r_{1}, \ldots, r_{k}\right)
$$

and

$$
I\left(n_{1}, \ldots, n_{k}\right):=\int_{n_{1}}^{n_{1}+1} \cdots \int_{n_{k}}^{n_{k}+1} \frac{\partial}{\partial u_{1}} \cdots \frac{\partial}{\partial u_{k}} g\left(u_{1} \sqrt{t}, \ldots, u_{k} \sqrt{t}\right) d u_{1} \cdots d u_{k} .
$$

By assumption $\left|W\left(n_{1}, \ldots, n_{k}\right)\right|<B$ (vanishing unless $n_{i} \geq N_{i}$ ), and hence

$$
\begin{aligned}
S & \leq B \int_{N_{1}}^{\infty} \cdots \int_{N_{k}}^{\infty}\left|\frac{\partial}{\partial u_{1}} \cdots \frac{\partial}{\partial u_{k}} g\left(u_{1} \sqrt{t}, \ldots, u_{k} \sqrt{t}\right)\right| d u_{1} \ldots d u_{k} \\
& =B \int_{N_{1} \sqrt{t}}^{\infty} \cdots \int_{N_{k} \sqrt{t}}^{\infty}\left|\frac{\partial}{\partial u_{1}} \cdots \frac{\partial}{\partial u_{k}} g\left(u_{1}, \ldots, u_{k}\right)\right| d u_{1} \ldots d u_{k} .
\end{aligned}
$$

Now since $Q\left(x_{1}, \ldots, x_{k}\right)$ is positive definite, we have

$$
Q\left(x_{1}, \ldots, x_{k}\right)>2 \lambda\left(x_{1}^{2}+\cdots+x_{k}^{2}\right)
$$

for some $\lambda>0$, giving

$$
\begin{aligned}
\left|\frac{\partial}{\partial u_{1}} \cdots \frac{\partial}{\partial u_{k}} g\left(u_{1}, \ldots, u_{k}\right)\right| & \leq\left|P\left(u_{1}, \ldots, u_{k}\right)\right| e^{-2 \lambda\left(u_{1}^{2}+\cdots+u_{k}^{2}\right)} \\
& =O\left(e^{-\lambda\left(u_{1}^{2}+\cdots+u_{k}^{2}\right)}\right)
\end{aligned}
$$

where $P\left(u_{1}, \ldots, u_{k}\right)=P_{Q}\left(u_{1}, \ldots, u_{k}\right)$ is some polynomial of total degree $k$. Thus

$$
S=\int_{N_{1} \sqrt{t}}^{\infty} \cdots \int_{N_{k} \sqrt{t}}^{\infty} O\left(e^{-\lambda\left(u_{1}^{2}+\cdots+u_{k}^{2}\right)}\right) d u_{1} \ldots d u_{k}=O\left(e^{-\lambda\left(N_{1}^{2}+\cdots+N_{k}^{2}\right) t}\right),
$$

as claimed.

Observing that (replacing $u_{i}$ by $-u_{i}$ as necessary) $T\left(e^{-t}\right)-q(0, \ldots, 0)$ can be written as a sum of sums of the form $S$ with at least one of the $N_{i} \geq 1$, and $T\left(e^{-t}\right)-T_{\vec{m}}\left(e^{-\lambda t}\right)$ as a sum of sums with at least one $N_{i} \geq m=\min m_{i}$, we have

$$
T\left(e^{-t}\right)-q(0, \ldots, 0)=O\left(e^{-\lambda t}\right), \quad T\left(e^{-t}\right)-T_{\bar{m}}\left(e^{-t}\right)=O\left(e^{-\lambda m^{2} t}\right) .
$$


Hence

$$
\begin{aligned}
F(s) & =\Gamma^{-1}(s) \int_{0}^{\infty}\left(T\left(e^{-t}\right)-q(0, \ldots, 0)\right) t^{s-1} d t \\
& =O\left(\left|\Gamma^{-1}(s)\right| \int_{0}^{\infty} e^{-\lambda t} t^{\sigma-1} d t\right)=O\left(\frac{\Gamma(\sigma)}{|\Gamma(s)|}\right)
\end{aligned}
$$

exists, and similarly

$$
\left|F(s)-A_{\vec{m}}(s)\right|=O\left(\left|\Gamma^{-1}(s)\right| \int_{0}^{\infty} e^{-\lambda m^{2} t} t^{\sigma-1} d t\right)=O\left(m^{-2 \sigma} \frac{\Gamma(\sigma)}{|\Gamma(s)|}\right) \rightarrow 0
$$

as $m \rightarrow \infty$ in any region $\{s: \sigma>\varepsilon,|s| \leq K\}$ for a fixed positive $\varepsilon$ and $K$. Thus the limit exists and is analytic for all $\operatorname{Re} s>0$.

Proof of the $\S 5$ formulae. We recall the $\theta$-functions

$$
\theta_{2}(q):=\sum_{n=-\infty}^{\infty} q^{(n+1 / 2)^{2}}, \quad \theta_{3}(q):=\sum_{n=-\infty}^{\infty} q^{n^{2}},
$$

and observe (as may be deduced from Zucker-Robertson [21, 22]) that when $\mathbf{Q}(\sqrt{-p})$ is a unique factorisation domain containing $u$ units

$$
\sum_{n=1}^{\infty} r\left(n, Q_{p}\right) q^{n}:=\theta_{2}(q) \theta_{2}\left(q^{p}\right)+\theta_{3}(q) \theta_{3}\left(q^{p}\right)=1+u \sum_{n=1}^{\infty}(-p \mid n) \frac{q^{n}}{\left(1-q^{n}\right)} .
$$

Hence $r\left(n, Q_{p}\right)$, the number of integer representations of $n$ by $Q_{p}(x, y)$, satisfies

$$
r\left(n, Q_{p}\right)=u \sum_{d \mid n}(-p \mid d),
$$

and (2) is plain.

When $p=3$ or 7 we can relate $r(n, p)$, the number of integer solutions of $x^{2}+$ $p y^{2}=n$, to $r\left(n, Q_{p}\right)$. Setting $N(x+y \sqrt{-p})=x^{2}+p y^{2}$ and writing

$$
Q_{p}(x, y)=\frac{1}{4}(x-y)^{2}+\frac{1}{4} p(x+y)^{2},
$$

it is easily seen that $r(n, p)$ represents the number of integer solutions $(x, y)$ of $N(x+y \sqrt{-p})=n$, while $r\left(n, Q_{p}\right)$ represents the number of integer solutions $(X, Y)$ of $N((X / 2)+(Y / 2) \sqrt{-p})=n$ with $X$ and $Y$ of the same parity. It is easily seen (matching $(x, y)=(X, Y))$ that $r(4 n, p)=r\left(n, Q_{p}\right)$, and by congruences modulo 4 that $r(n, p)=0$ when $n \equiv 2(\bmod 4)$. When $n$ is odd and $p=7$, congruences modulo 8 show that if $N((X / 2)+(Y / 2) \sqrt{-p})=n$ then $X$ and $Y$ are necessarily even (giving a pairing $(x, y)$ and $(X / 2, Y / 2))$, and $r(n, 7)=r\left(n, Q_{7}\right)$. When $n$ is odd and $p=3$, putting $\omega=(1+\sqrt{-3}) / 2$, it is readily checked that exactly one of the $r\left(n, Q_{3}\right)$ solutions $((X / 2)+(Y / 2) \sqrt{-3}),((X / 2)+(Y / 2) \sqrt{-3}) \omega$ and $((X / 2)+(Y / 2) \sqrt{-3}) \omega^{2}$ will be of the form $(x+y \sqrt{-p})$, and hence that $r(n, 3)=\frac{1}{3} r\left(n, Q_{3}\right)$.

Thus, setting $\lambda=1$ or $1 / 3$ as $p=7$ or 3 respectively, we have

$$
\begin{aligned}
\sum_{n=1}^{\infty} \frac{( \pm 1)^{n} r(n, p)}{n^{s}} & =\sum_{n=1}^{\infty} \frac{r\left(n, Q_{p}\right)}{(4 n)^{s}} \pm \lambda \sum_{\substack{n=1 \\
n \text { odd }}}^{\infty} \frac{r\left(n, Q_{p}\right)}{n^{s}} \\
& =\left(4^{-s} \pm \lambda\left(1-2^{-s}\right)\left(1-(-p \mid 2) 2^{-s}\right)\right)\left(\sum_{n=1}^{\infty} \frac{r\left(n, Q_{p}\right)}{n^{s}}\right) .
\end{aligned}
$$


So finally, noting that $(-3 \mid 2)=-1,(-7 \mid 2)=1$ and $\left(1-2^{1-s}\right) \zeta(s)=\alpha(s)$, we have

$$
\begin{gathered}
\sum_{(x, y) \in \mathbf{Z}^{\backslash} \backslash(0,0)} \frac{1}{\left(x^{2}+3 y^{2}\right)^{s}}=2\left(1+2^{1-2 s}\right) \zeta(s) L_{-3}(s), \\
\sum_{(x, y) \in \mathbf{Z}^{2} \backslash(0,0)} \frac{(-1)^{x+y}}{\left(x^{2}+3 y^{2}\right)^{s}}=-2\left(1+2^{1-s}\right) \alpha(s) L_{-3}(s), \\
\sum_{(x, y) \in \mathbf{Z}^{\backslash} \backslash(0,0)} \frac{1}{\left(x^{2}+7 y^{2}\right)^{s}}=2\left(1-2^{1-s}+2^{1-2 s}\right) \zeta(s) L_{-7}(s), \\
\sum_{(x, y) \in \mathbf{Z}^{\backslash} \backslash(0,0)} \frac{(-1)^{x+y}}{\left(x^{2}+7 y^{2}\right)^{s}}=-2 \alpha(s) L_{-7}(s) .
\end{gathered}
$$

\section{REFERENCES}

1. D. Borwein, J. M. Borwein \& K. Taylor, Convergence of lattice sums and Madelung's constant, J. Math. Phys. 26 (1985), 2999-3009. MR 86m:82047

2. J. M. Borwein \& P. B. Borwein, Pi and the AGM - A Study in Analytic Number Theory and Computational Complexity, Wiley, New York, 1987. MR 89a:11134

3. D. Borwein, J.M. Borwein, \& R. Shail, Analysis of certain lattice sums, J. Math. Anal. Appl. 143 (1989), 126-137. MR 90j:82038

4. J. P. Buhler \& R. E. Crandall, On the convergence problem for lattice sums, J. Phys. A: Math. Gen. 23 (1990), 2523-2528. MR 91h:82008

5. M. L. Glasser \& I. J. Zucker, Lattice Sums, Theoret. Chem. Adv. \& Perspectives, 5 (1980), 67-139.

6. G. H. Hardy \& M. Riesz, The General Theory of Dirichlet Series, Cambridge Tracts in Mathematics and Mathematical Physics, Cambridge University Press, 1915.

7. M. N. Huxley, Exponential sums and lattice points II, Proc. London Math. Soc. 66 (1993), 279-301. MR 94b:11100

8. E. Krätzel, Bemerkungen zu einem Gitterpunktsproblem, Math. Ann. 179 (1969), 90-96. MR 39:4108

9. E. Krätzel \& W. Nowak, Lattice points in large convex bodies, II, Acta Arith. 62 (1992), 285-295. MR 93m:11102

10. E. Landau, Zur analytischen Zahlentheorie der definiten quadratischen Formen (über Gitterpunkte in mehrdimensionalen Ellipsoiden), S.B. Preuss. Akad. Wiss. (1915), 458-476.

11. E. Landau, Über Gitterpunkte in mehrdimensionalen Ellipsoiden, Math. Zeit. 21 (1924), 126132.

12. E. Landau, Über Gitterpunkte in mehrdimensionalen Ellipsoiden, Math. Zeit. 24 (1926), 299310.

13. E. Landau, Vorselungen über Zahlentheorie, zweiter Band, achter Teil, Kap 6. Chelsea Publishing Company, New York 1955.

14. B. Novák, Über eine Methode der $\Omega$-Abschätzungen, Czech. Math. J. 21 (1971), 257-279. MR 45:1843

15. B. Novák, New proofs of a theorem of Edmund Landau, Acta Arith. 31 (1976), 101-105. MR 54:7413

16. M. Riesz, Sur un théorème de la moyenne et ses applications, Acta Univ. Hungaricae Franc.Jos. 1 (1923), 114-126.

17. A. Walfisz, Über Gitterpunkte in mehrdimensionalen Ellipsoiden, Math. Zeit. 19 (1924), 300307.

18. A. Walfisz, Convergence abscissae of certain Dirichlet series, Akad. Nauk Gruzin. SSR. Trudy Tbiliss. Mat. Inst. Razmadze, 22 (1956), 33-75 (in Russian). MR 19:943f

19. G. N. Watson, A Treatise on the Theory of Bessel Functions, Cambridge University Press, 1922. MR 96i:33010 (later ed.) 
20. J. R. Wilton, A series of Bessel functions conected with the theory of lattice points, Proc. London Math. Soc. 29 (1928), 168-188.

21. I. J. Zucker \& M. M. Robertson, Some properties of Dirichlet L-series, J. Phys. A: Math. Gen. 9 (1976), 1207-1214. MR 54:253

22. I. J. Zucker \& M. M. Robertson, A systematic approach to the evaluation of $\sum_{(m, n \neq 0,0)}\left(a m^{2}+\right.$ bmn $\left.+c n^{2}\right)^{-s}$, J. Phys. A: Math. Gen. 9 (1976), 1215-1225. MR 54:244

Department of Mathematics, University of Western Ontario, London, Ontario N6A 5B7, CANADA

E-mail address: dborwein@uwo.ca

Centre for Experimental and Constructive Mathematics, Simon Fraser University, Burnaby, BC V5A 1S6, Canada

E-mail address: jborwein@cecm.sfu.ca

Centre for Experimental and Constructive Mathematics, Simon Fraser University, Burnaby, BC V5A 1S6, Canada \& Department of Mathematics, University of British Columbia, Vancouver, BC V6T 1Z2, Canada

E-mail address: pinner@cecm.sfu.ca 